\title{
Millimeter and submillimeter excess emission in M 33 revealed by Planck and LABOCA
}

\author{
I. Hermelo ${ }^{1}$, M. Relaño ${ }^{2,3}$, U. Lisenfeld ${ }^{2,3}$, S. Verley ${ }^{2,3}$, C. Kramer ${ }^{1}$, T. Ruiz-Lara ${ }^{2,3}$, M. Boquien ${ }^{4}$, \\ E. M. Xilouris ${ }^{5}$, and M. Albrecht ${ }^{6}$
}

\author{
${ }^{1}$ Instituto Radioastronomía Milimétrica (IRAM), Av. Divina Pastora 7, Núcleo Central, 18012 Granada, Spain \\ e-mail: hermelo@iram.es \\ 2 Departamento de Física Teórica y del Cosmos, Universidad de Granada, 18071 Granada, Spain \\ 3 Instituto Universitario Carlos I de Física Teórica y Computacional, Universidad de Granada, 18071 Granada, Spain \\ ${ }^{4}$ Unidad de Astronomía, Fac. Cs. Básicas, Universidad de Antofagasta, Avda. U. de Antofagasta 02800, Antofagasta, Chile \\ 5 Institute for Astronomy, Astrophysics, Space Applications \& Remote Sensing, National Observatory of Athens, P. Penteli, \\ 15236 Athens, Greece \\ 6 Argelander-Institut für Astronomie, Universität Bonn, 53121 Bonn, Germany
}

Received 5 February 2015 / Accepted 3 March 2016

\begin{abstract}
Context. Previous studies have shown the existence of an excess of emission at submillimeter (submm) and millimeter (mm) wavelengths in the spectral energy distribution (SED) of many low-metallicity galaxies. The so-called "submm excess", whose origin remains unknown, challenges our understanding of the dust properties in low-metallicity environments.

Aims. The goal of the present study is to model separately the emission from the star forming (SF) component and the emission from the diffuse interstellar medium (ISM) in the nearby spiral galaxy M33 in order to determine whether both components can be well fitted using radiation transfer models or whether there is an excess of submm emission associated with one or both of them.

Methods. We decomposed the observed SED of M 33 into its SF and diffuse components. Mid-infrared (MIR) and far-infrared (FIR) fluxes were extracted from Spitzer and Herschel data. At submm and mm wavelengths, we used ground-based observations from APEX to measure the emission from the SF component and data from the Planck space telescope to estimate the diffuse emission. Both components were separately fitted using radiation transfer models based on standard dust properties (i.e., emissivity index $\beta=2$ ) and a realistic geometry. The large number of previous studies helped us to estimate the thermal radio emission and to constrain an important part of the input parameters of the models. Both modeled SEDs were combined to build the global SED of M 33. In addition, the radiation field necessary to power the dust emission in our modeling was compared with observations from GALEX, Sloan, and Spitzer.

Results. Our modeling is able to reproduce the observations at MIR and FIR wavelengths, but we found a strong excess of emission at submm and $\mathrm{mm}$ wavelengths where the model expectations severely underestimate the LABOCA and Planck fluxes. We also found that the ultraviolet (UV) radiation escaping the galaxy is $70 \%$ higher than the model predictions. From the total mass of dust derived from our modeling and the mass of atomic and molecular gas measured with the VLA and the IRAM $30 \mathrm{~m}$ telescope, we determined a gas-to-dust mass ratio $G_{\text {dust }} \sim 100$, significantly lower than the value expected from the subsolar metallicity of M 33 .

Conclusions. We discussed different hypotheses to explain the discrepancies found in our study (i.e., excess of emission at submm and $\mathrm{mm}$ wavelengths, deficit of UV attenuation, and abnormally low value of $G_{\text {dust }}$ ), concluding that different dust properties in M 33 is the most plausible explanation.
\end{abstract}

Key words. dust, extinction - galaxies: ISM - galaxies: individual: M 33 - galaxies: star formation - submillimeter: galaxies

\section{Introduction}

Dust grains are mixed with the gas in all the components and phases of a galaxy, from the ionized gas and the photodissociation regions (PDRs) embedded in the star forming (SF) regions to the diffuse interstellar medium (ISM). Depending on their environment, dust grains are exposed to very different radiation fields and physical conditions. While the interstellar dust within the SF component is immersed in the intense ultraviolet (UV) radiation coming from massive hot stars, dust grains in the diffuse ISM are more likely illuminated by the radiation field of the old stellar population (Hippelein et al. 2003; Verley et al. 2009). These differences not only change the grain heating and the subsequent emission, but might also change the grain properties (e.g., size distribution, chemical composition, ionization state) and play an important role in dust evolution (Hippelein et al. 2003; Paradis et al. 2011).

Modeling the spectral energy distribution (SED) emitted by the dust grains is a powerful tool for understanding the grain properties as well as the interaction between the interstellar dust and the radiation field. One of the simplest approaches to modeling the dust emission is the modified black body (MBB), where the intensity, $S_{v}$, is expressed as

$S_{v} \propto B_{v}(T) v^{\beta}$,

where $B_{v}(T)$ is the Planck function and $\beta$ is the dust emissivity index, which in the case of idealized spherical dielectric grains adopts the value $\beta=2$. Although the MBB models are highly degenerated (there is no unique solution for $\beta$ and $T$, see Tabatabaei et al. 2014, for a detailed analysis), they have been 
widely used to study dust emission (e.g., Kirkpatrick et al. 2013; Gordon et al. 2014).

More sophisticated models have also been used, from phenomenological models based on realistic dust properties (e.g., Galliano et al. 2011) to fully three-dimensional radiation transfer models (Ercolano et al. 2005). Although the different methodologies applied make the comparison of these studies somewhat difficult, many of them have shown that it is not possible to reproduce the SEDs of low-metallicity galaxies owing to an excess of observed emission at submillimeter (submm) wavelengths (e.g., Lisenfeld et al. 2002; Galliano et al. 2003, 2005; Bendo et al. 2006; Galametz et al. 2009, 2011; Israel et al. 2010; Bot et al. 2010; Dale et al. 2012; Planck Collaboration XVII 2011).

The origin of this problem, usually known as the submm excess, is not clear. The existence of a large amount of cold dust could, in principle, produce the observed shape, but the required dust mass is unreasonably high (e.g., Lisenfeld et al. 2002) and the gas-to-dust mass ratio too low (e.g., Galliano et al. 2011; Galametz et al. 2011; Gordon et al. 2014). Different dust grain properties, and in particular a lower $\beta$ in the submm have also been suggested (e.g., Lisenfeld et al. 2002; Galliano et al. 2011; Gordon et al. 2014). For example, if hydrogenated amorphous carbon grains were the most probable form of carbonaceous grains in the ISM (instead of graphite), this would significantly flatten the SED in the submm range (Serra Díaz-Cano \& Jones 2008; Jones et al. 2013). More exotic explanations have also been proposed, from magnetic nanograins emitting at the microwave and submm wavelengths (Draine \& Hensley 2012) to spinning grains (Draine \& Lazarian 1998), which emit instead in the millimeter ( $\mathrm{mm}$ ) range (Bot et al. 2010).

A step forward has been the possibility of carrying out spatially resolved analysis of the dust SED with a combination of Spitzer and Herschel data, which allow the investigation of variations of the dust properties at small scales. The Magellanic Clouds and M 33 are excellent targets for performing such studies. In the Large Magellanic Cloud (LMC) Galliano et al. (2011) found evidence for a submm excess in the low surface brightness areas by fitting the dust SED locally with a detailed dust model. Gordon et al. (2014) were able to fit the SEDs of the LMC and the Small Magellanic Cloud (SMC) using a MBB with a broken powerlaw for $\beta$. For M 33 Tabatabaei et al. (2014) carried out a MBB fit of the local SED and found a trend of decreasing temperature $T$ and decreasing $\beta$ with radius (the opposite of what would be expected from the $\beta$ - $T$ degeneracy). They concluded that a change in the dust properties is the most likely reason for this trend. Other studies have tried to look for variations in the dust properties in additional objects. Galametz et al. (2014) investigated the $870 \mu \mathrm{m}$ emission of a sample of 11 nearby galaxies and found evidence for a submm excess in 4 of them. For three of these objects the excess was in the outskirts of the galaxy. Hermelo et al. (2013) decomposed the observed emission of the dwarf, low-metallicity galaxy NGC 4214 into its SF and diffuse components. They modeled these components separately using radiation transfer models and found indications of submm excess in the diffuse component of NGC 4214.

This paper is part of a series of studies that use radiation transfer models to fit the SED of a sample of dwarf starburst and late-type spiral disk galaxies. Here we apply the same methodology as in Hermelo et al. (2013) to the Local Group galaxy M 33. The proximity of M33 (0.840 Mpc, Freedman et al. 1991), its moderate inclination angle (57 $7^{\circ}$, Deul \& van der Hulst 1987), and the wide data set available make this galaxy an excellent target to study separately the SEDs of the SF and the diffuse component with accuracy. Recent results of the open time key program HerM33es (Kramer et al. 2010) have shown that the Herschel observations of M33 cannot be well reproduced assuming standard dust properties (i.e., MBB model with $\beta=2$ ). Instead, these authors had to adopt a MBB model with $\beta=1.5$ to obtain a good fit. The value of $\beta=1.5$ is extendible to the Planck data (see Appendix A for details) and suggests that the dust properties or conditions might be special in M 33 .

The aim of the present paper is to investigate whether it is possible to reproduce the SED of M 33 with standard dust grain properties but using more sophisticated models based on radiation transfer calculations and a realistic geometry, or whether the anomalies found by Kramer et al. (2010) persist. The paper is organized as follows. In Sect. 2 we present the data set used, from UV to radio. Section 3 is devoted to explaining the photometry measurements and the method we used to separate the emission coming from the SF regions and the ISM in the galaxy. In Sect. 4 we explain briefly the models applied to fit the SED of the two components and how we determined their input parameters. The results of the modeling are presented in Sect. 5. In Sect. 6 we discuss the results and in Sect. 7 we summarize the main conclusions of this paper.

\section{Data}

A wide range of data from recent scientific missions is available for M 33 in archives. In this section we describe the data used in our study, which are summarized in Table 1.

\subsection{GALEX}

To investigate the continuum UV emission associated with the young stellar population of M33, we used the data from the Galaxy Evolution Explorer (GALEX, Martin et al. 2005) distributed by Gil de Paz et al. (2007). The GALEX far-UV (FUV $\sim 154 \mathrm{~nm}$ ) and near-UV (NUV $\sim 232 \mathrm{~nm}$ ) observations and the data reduction can be found in Thilker et al. (2005). The angular resolution for the GALEX FUV and NUV maps is $4 . \prime 2$ and 5'!3, respectively. Following Morrissey et al. (2007), we conservatively adopted a calibration error of $10 \%$.

\subsection{Sloan}

We used data from the Sloan Digital Sky Survey (SDSS, York et al. 2000) to measure the stellar emission in the $u$-band and to calculate the $b$-band stellar disk scale-length of M33. Owing to the small field of view (FoV) of the SDSS images, we needed to reconstruct a mosaic to cover the whole extension of M33 in these bands. For this purpose we used the software Montage ${ }^{1}$. Montage is a toolkit for creating mosaics from individual Flexible Image Transport System (FITS) images. We made use of the data from the seventh data release (DR7, Abazajian et al. 2009). These images have a spatial coverage of $13.51 \times 9.83$, an exposure time of $53.9 \mathrm{~s}$, and the angular resolution is $\sim 1^{\prime \prime} .4$ (median value measured in the Sloan $r$-band). The SDSS DR7 pipeline does not apply any sky subtraction to the raw data. Considering that most of the frames used to reconstruct the mosaics are fully covered by light coming from M 33, using the DR7 data allows us to avoid possible artifacts coming from an inaccurate sky subtraction. Following Padmanabhan et al. (2008) we adopted a calibration error of $1 \%$ for the Sloan $g$-band and $2 \%$ for the Sloan $u$-band.

\footnotetext{
1 http://montage.ipac. caltech.edu
} 
Table 1. Summary of the observations used in this study.

\begin{tabular}{|c|c|c|c|c|c|c|c|}
\hline Telescope & $\begin{array}{l}\text { Instrument } \\
\text { or filter }\end{array}$ & $\begin{array}{c}\lambda_{0} \\
\mu \mathrm{m}\end{array}$ & $\begin{array}{c}v_{0} \\
\mathrm{GHz}\end{array}$ & $F W H M$ & $\begin{array}{c}\Delta_{\mathrm{CAL}} \\
\% \\
\end{array}$ & Band name & References \\
\hline \multirow[t]{2}{*}{ GALEX } & FUV & 0.154 & 1946704 & $4 ! 2$ & 10 & GALEX FUV & Thilker et al. (2005) \\
\hline & NUV & 0.232 & 1292209 & $5 ! 3$ & 10 & GALEX NUV & Thilker et al. (2005) \\
\hline \multirow[t]{2}{*}{ Sloan } & $u$ & 0.355 & 844486 & $1 " 44$ & 2 & Sloan $u$-band & This paper. \\
\hline & $g$ & 0.469 & 639216 & $1 " 44$ & 1 & Sloan $g$-band & This paper. \\
\hline KPNO & $\mathrm{H} \alpha$ & 0.657 & 456305 & $6 " .6$ & 15 & $\mathrm{H} \alpha$ & Greenawalt (1998) \\
\hline \multirow[t]{3}{*}{ Spitzer } & IRAC & 3.55 & 84449 & $2 \prime .5$ & 2 & IRAC $3.6 \mu \mathrm{m}$ & Verley et al. (2007) \\
\hline & IRAC & 4.49 & 66769 & $2 " 9$ & 2 & IRAC $4.5 \mu \mathrm{m}$ & Verley et al. (2007) \\
\hline & MIPS & 23.7 & 12650 & $6 " 3$ & 4 & MIPS $24 \mu \mathrm{m}$ & This paper. \\
\hline \multirow[t]{6}{*}{ Herschel } & PACS & 70.0 & 4283 & $5 \prime 66$ & 10 & PACS $70 \mu \mathrm{m}$ & Boquien et al. (2015) \\
\hline & PACS & 100 & 2998 & $9 ": 8$ & 10 & PACS $100 \mu \mathrm{m}$ & Boquien et al. (2011) \\
\hline & PACS & 160 & 1874 & $13 " .6$ & 20 & PACS $160 \mu \mathrm{m}$ & Boquien et al. (2011) \\
\hline & SPIRE & 250 & 1199 & $18 \prime \prime 1$ & 5 & SPIRE $250 \mu \mathrm{m}$ & Xilouris et al. (2012) \\
\hline & SPIRE & 363 & 826 & $24 \prime \prime 9$ & 5 & SPIRE $350 \mu \mathrm{m}$ & Xilouris et al. (2012) \\
\hline & SPIRE & 517 & 580 & $36 \prime .4$ & 5 & SPIRE $500 \mu \mathrm{m}$ & Xilouris et al. (2012) \\
\hline APEX & LABOCA & 870 & 345 & $19 \prime 22$ & 10 & LABOCA $870 \mu \mathrm{m}$ & Albrecht et al. (in prep.) \\
\hline \multirow[t]{7}{*}{ Planck } & HFI & 350 & 857 & 4.63 & 10 & PLANCK $350 \mu \mathrm{m}$ & This paper. \\
\hline & HFI & 550 & 545 & $4 ! 84$ & 10 & PLANCK $550 \mu \mathrm{m}$ & This paper. \\
\hline & HFI & 850 & 353 & $4 ! 86$ & 3 & PLANCK $850 \mu \mathrm{m}$ & This paper. \\
\hline & HFI & 1380 & 217 & $5 \vdots 01$ & 3 & PLANCK $1.4 \mathrm{~mm}$ & This paper. \\
\hline & HFI & 2096 & 143 & $7: 27$ & 3 & PLANCK $2.1 \mathrm{~mm}$ & This paper. \\
\hline & HFI & 3000 & 100 & $9 ! 66$ & 3 & PLANCK $3.0 \mathrm{~mm}$ & This paper. \\
\hline & LFI & 10000 & 30 & $32: 3$ & 3 & PLANCK $10 \mathrm{~mm}$ & This paper. \\
\hline Effelsberg & $\mathrm{S} 36 \mathrm{~mm}$ & 35903 & 8.35 & $1: 39$ & $*$ & $\mathrm{~S} 36 \mathrm{~mm}$ & Tabatabaei et al. (2007b) \\
\hline
\end{tabular}

Notes. ${ }^{(*)}$ Only the total error is reported in Tabatabaei et al. (2007b).

\section{3. $H \alpha$}

To trace the ionized gas, we used the narrow band $\mathrm{H} \alpha$ image of M 33 obtained with the Kitt Peak National Observatory (KPNO) by Greenawalt (1998). The reduction process uses standard IRAF ${ }^{2}$ procedures to subtract the continuum emission and is described in detail in Hoopes \& Walterbos (2000). The total FoV of the image is $1.75 \times 1.75 \mathrm{deg}^{2}(2048 \times 2048$ pixels with a pixel scale of 2 ".03) with a $66^{\prime \prime} 6$ resolution. We consider typical uncertainties to be better than $15 \%$ for the $\mathrm{H} \alpha$ photometric measurements (Verley et al. 2010b).

\subsection{Spitzer}

Data for M 33 was obtained with the Spitzer (Werner et al. 2004) Infrared Array Camera (IRAC, Fazio et al. 2004) and the Multiband Imaging Photometer (MIPS, Rieke et al. 2004). We used IRAC $3.6 \mu \mathrm{m}$ and $4.5 \mu \mathrm{m}$ data to study the radiation field associated with the old stellar population of M 33 and MIPS $24 \mu \mathrm{m}$ to study the dust emission. The MIPS $70 \mu \mathrm{m}$ and MIPS $160 \mu \mathrm{m}$ data were not used in this work since better quality data from PACS are available at the same wavelengths. The emission from polycyclic aromatic hydrocarbons (PAHs), traced by the

2 IRAF is distributed by the National Optical Astronomy Observatories, which are operated by the Association of Universities for Research in Astronomy, Inc., under cooperative agreement with the National Science Foundation.
IRAC $5.8 \mu \mathrm{m}$ and $8.0 \mu \mathrm{m}$ bands, is out of the scope of the present work.

IRAC $3.6 \mu \mathrm{m}$ and $4.5 \mu \mathrm{m}$ data are described in Verley et al. (2007, 2009, 2010a). The MIPS $24 \mu \mathrm{m}$ data were processed using version 18.5 of the MOPEX software (MOsaicker and Point source EXtractor, Makovoz \& Marleau 2005) to combine the 16 Astronomical Observation Requests (AORs) available in the data archive. The spatial resolution is $2^{\prime \prime} 5,22^{\prime \prime} 9$, and 6".3 for the IRAC $3.6 \mu \mathrm{m}$, IRAC $4.5 \mu \mathrm{m}$, and MIPS $24 \mu \mathrm{m}$ bands, respectively. We adopted calibration errors of $2 \%$ and for the IRAC bands (Reach et al. 2005) and 4\% for MIPS $24 \mu \mathrm{m}$ (Engelbracht et al. 2007).

\subsection{Herschel}

As part of the HerM33es key program (Kramer et al. 2010), the M33 galaxy was mapped by Herschel with the Photodetector Array Camera and Spectrometer (PACS, Poglitsch et al. 2010) and the Spectral and Photometric Imaging Receiver (SPIRE, Griffin et al. 2010). PACS $100 \mu \mathrm{m}$ and $160 \mu \mathrm{m}$ and SPIRE $250 \mu \mathrm{m}, 350 \mu \mathrm{m}$, and $500 \mu \mathrm{m}$ observations were done in parallel mode in two orthogonal directions on January 7, 2010 , covering a region of about $1.36 \mathrm{sq}$. deg. The PACS $70 \mu \mathrm{m}$ image was obtained as a follow-up open time cycle 2 programme, on 25 June 2012 in two orthogonal directions and five repetitions to achieve better sensitivity (Boquien et al. 2015). The PACS reduction was performed using the mapmaking software Scanamorphos (Roussel 2013) as described 
in Boquien et al. (2011). The SPIRE reduction was done using the Herschel Data Processing System (HIPE, Ott 2010, 2011) and the maps were created using a "naive" mapping projection (Verley et al. 2010b; Boquien et al. 2010; Xilouris et al. 2012). The angular resolution of the Herschel data range from 5".6 at $70 \mu \mathrm{m}$ to 36 ". 4 for the $500 \mu \mathrm{m}$ band. The absolute photometric uncertainty is $10 \%$ for the PACS $70 \mu \mathrm{m}$ and $100 \mu \mathrm{m}$ bands and $20 \%$ for the PACS $160 \mu \mathrm{m}$ band (Poglitsch et al. 2010), while we adopted $5 \%$ as the calibration uncertainty for the three SPIRE bands (Bendo et al. 2013).

\section{6. $\angle A B O C A$}

Observations of M33 were carried out at the Atacama Pathfinder Experiment (APEX, Güsten et al. 2006) using the Large Bolometer Camera (LABOCA, Siringo et al. 2009) between 2010 and 2012 (Albrecht et al., in prep.). A filter set coupled to the atmospheric window provides a bandwidth of $\sim 150 \mu \mathrm{m}(\sim 60 \mathrm{GHz})$ around the central frequency of $870 \mu \mathrm{m}$ $(345 \mathrm{GHz})$. From observations of Uranus an almost circular beam size of $19^{\prime \prime} .2 \pm 0$ 0'.7 was determined (Siringo et al. 2009). LABOCA mapping was performed in on-the-fly mode parallel and orthogonal to the major axis of M33. Flux calibration was achieved through observations of planets and a set of secondary calibrators and was found to be accurate within $10 \%$ (Siringo et al. 2009). To enable the usage of the LABOCA data in multiwavelength studies the flux calibration methods for LABOCA and SPIRE were compared to ensure a correct crosscalibration. On average, flux densities from the LABOCA calibration were found to be $2 \%$ lower than the SPIRE method.

The LABOCA data were reduced using the $\mathrm{BoA}^{3}$ software package. The applied skynoise suppression can not distinguish between correlated atmospheric noise and astronomical emission that is uniformly extended over areas equal to or larger than the area covered by the groups of bolometer channels (defined as having the same amplifier box or the same wiring) that are used to derive the median noise. This typically filters out uniform emission on scales larger than about 2.5 (for details see Schuller et al. 2009). To minimize the effect an iterative approach by subsequently improving a model of the flux distribution was applied. Nevertheless, given the large apparent size of M33, the resulting LABOCA map does not recover the entire contribution from the extended emission, as is confirmed by comparing it with the corresponding PLANCK $850 \mu \mathrm{m}$ map (see Fig. 1).

\subsection{Planck}

We used Planck data from the 2013 distribution of released products (Planck Collaboration I 2014) based on the data acquired during the Planck nominal operations period, i.e., between 12 August 2009 and 27 November 2010. Data can be accessed via the Planck Legacy Archive ${ }^{4}$.

In the case of the Low Frequency Instrument (LFI) $10 \mathrm{~mm}$ $(30 \mathrm{GHz})$ band, we used the flux computed in the Planck Catalogue of Compact Sources (Planck Collaboration XXVIII 2014). For the other Planck bands, i.e., the High Frequency Instrument (HFI) $350 \mu \mathrm{m}(857 \mathrm{GHz}), 550 \mu \mathrm{m}(545 \mathrm{GHz}), 850 \mu \mathrm{m}$ $(353 \mathrm{GHz}), 1.4 \mathrm{~mm}(217 \mathrm{GHz}), 2.1 \mathrm{~mm}(143 \mathrm{GHz})$, and $3.0 \mathrm{~mm}$ $(100 \mathrm{GHz})$ bands and the LFI $4.3 \mathrm{~mm}(70 \mathrm{GHz})$ and $6.8 \mathrm{~mm}$

\footnotetext{
3 http://www .astro.uni-bonn.de/boawiki/Boa

4 http://www. sciops.esa.int/index.php?project= planck\&page=Planck_Legacy_Archive
}

(44 GHz) bands, we extracted the fluxes from the Planck AllSky maps. These maps consist of 2048 HEALPix full-sky maps (Górski et al. 2005) corresponding to a pixel size of 2!64 with an angular resolution that ranges from 4.63 at $350 \mu \mathrm{m}$ to $32: 3$ at $10 \mathrm{~mm}$ (Planck Collaboration XXVIII 2015). To generate a Gnomonic projection of the HEALPix data centered on M33 we made use of the IDL facility Gnomview ${ }^{5}$. The cosmic microwave background (CMB) was subtracted from all the bands (see Appendix B for details). The contribution from other components (e.g., cirrus, cosmic infrared background) to the background was subtracted as the median value in a $5^{\prime}$ width annulus enclosing M33. The resulting maps are shown in Fig. 1. M33 was not detected in the $4.3 \mathrm{~mm}$ and $6.8 \mathrm{~mm}$ channels. We adopted a $\pm 10 \%$ calibration uncertainty for the $350 \mu \mathrm{m}$ and $550 \mu \mathrm{m}$ bands and $\pm 3 \%$ for all the other wavelengths (Planck Collaboration XXVIII 2015).

\subsection{Effelsberg}

Tabatabaei et al. (2007a) studied the emission at $3.6 \mathrm{~cm}$ $(8.35 \mathrm{GHz})$ of $\mathrm{M} 33$. Here we used their results to constrain the thermal radio emission of the SF regions and to decontaminate our measurements from synchrotron emission (see Sect. 3.3 for details).

They found a total flux of $761 \pm 63 \mathrm{mJy}$ for the inner $7.5 \mathrm{kpc}$ and a thermal fraction of $51.4 \pm 4.2 \%$ at this wavelength. This fraction was obtained from the extinction corrected $\mathrm{H} \alpha$ map of the galaxy, which was derived using a map of optical depth at $160 \mu \mathrm{m}$ and an extinction law for a standard dust model for the diffuse emission. The authors also derived the thermal fraction using the classical method and assuming a constant non-thermal spectral index for the whole galaxy of $\alpha_{n}=1.0 \pm 0.1$. With this method they obtained a thermal fraction of $63.2 \pm 5.3 \%$.

As a compromise between the two values of the thermal fraction derived by Tabatabaei et al. (2007a), we adopted the mean value as the best estimate and we allowed it to fluctuate between the lowest and the highest possible values. We therefore estimated that $57.3 \pm 11.2 \%(436 \pm 49 \mathrm{mJy})$ of the total flux at $8.35 \mathrm{GHz}$ corresponds to thermal radio emission.

\section{Photometry}

Prior to performing the photometry, we first homogenized all the images to the same data unit in order to measure all fluxes in Jy/pixel (with a pixel size of $14^{\prime \prime}$ ). Bright foreground stars were removed from the UV and optical data. We then convolved all the data to the resolution of SPIRE $500 \mu \mathrm{m}$ using the set of kernels provided by Aniano et al. $(2011)^{6}$; the only exception was the Planck data, which have a coarser resolution than the SPIRE $500 \mu \mathrm{m}$ image, and so they were only used to obtain the total photometry of M33. Using the IRAF task wregister, we then regridded all the images to the pixel size of the SPIRE $500 \mu \mathrm{m}$ map. Color corrections were applied to all the $\mathrm{MIR} / \mathrm{FIR} / \mathrm{submm} / \mathrm{mm}$ fluxes (see Appendix A for details). In this section we describe how we separated the different components, how we corrected our measurements from undesired contributions (e.g., CO line emission), and how the errors were handled.

\footnotetext{
5 http://healpix.jpl.nasa.gov/html/idlnode21.htm

6 http://www .astro.princeton. edu/ ganiano/Kernels.html
} 
I. Hermelo et al.: Millimeter and submillimeter excess emission in M 33 revealed by Planck and LABOCA

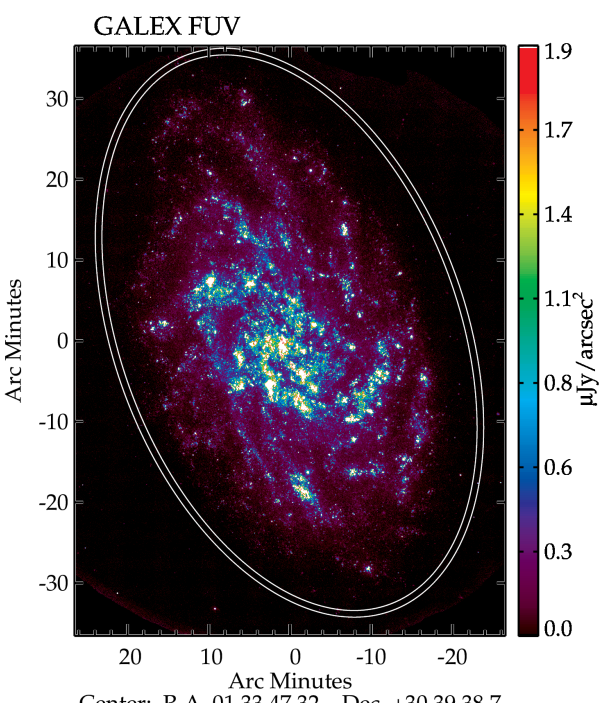

Center: R.A. 013347.32 Dec +303938.7

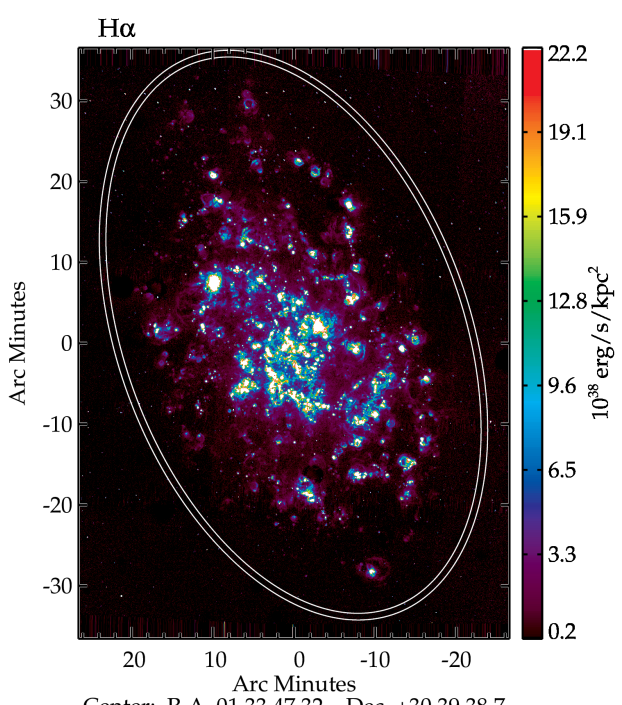

Center: R.A. 013347.32 Dec +303938.7

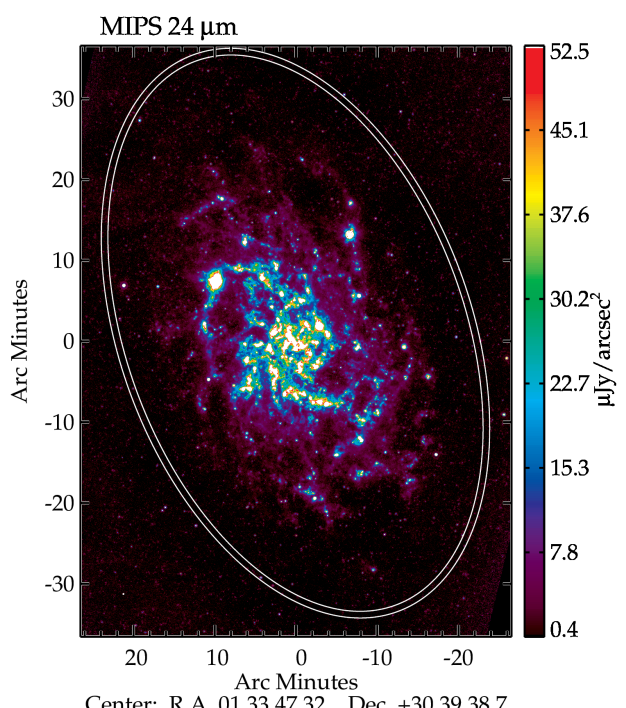

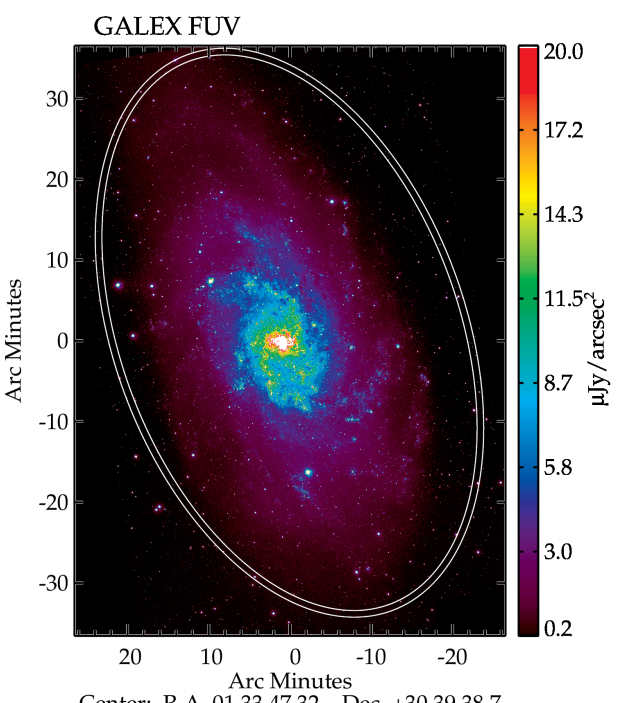

Center: R.A. 013347.32 Dec +303938.7

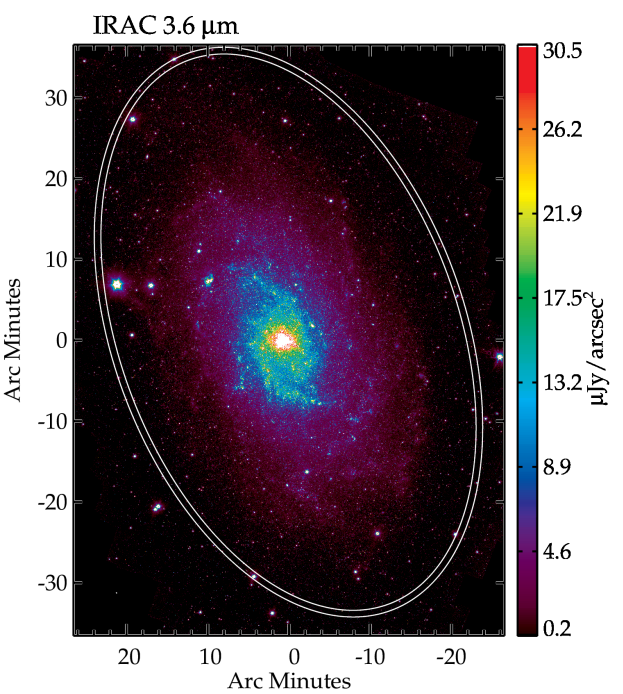

Center: R.A. 013347.32 Dec +303938.7

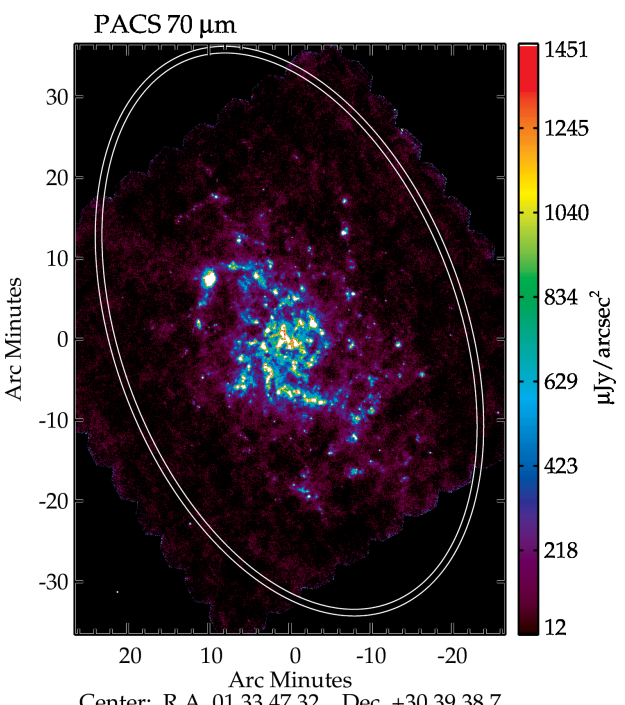

Fig. 1. Data of M 33 at their original resolution. Units are homogenized to facilitate the comparison between the different bands. At the distance of M 33, 1" subtends $\sim 4 \mathrm{pc}$. The white annulus, used to subtract the local background, is included for reference. The white circle at the bottom left (too tiny to be visible for bands with $\lambda \lesssim 350 \mu \mathrm{m}$ ) corresponds to the beam size. 


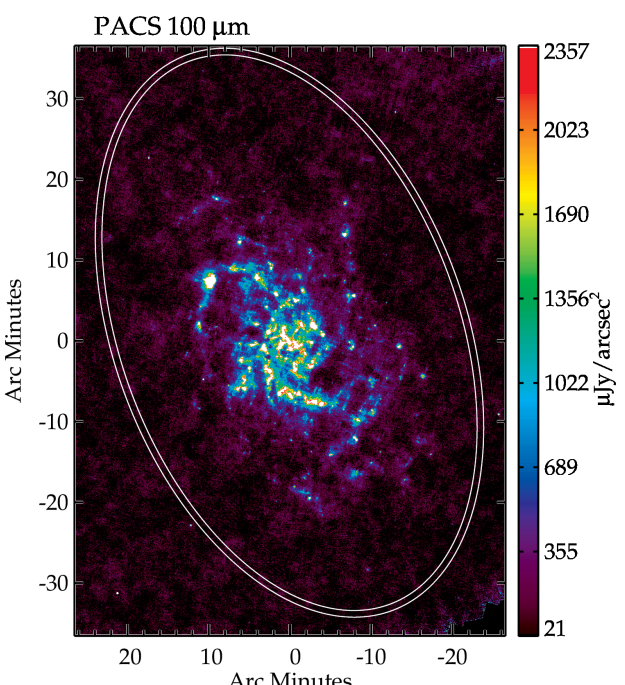

Center: R.A. 013347.32 Dec +30 3938.7

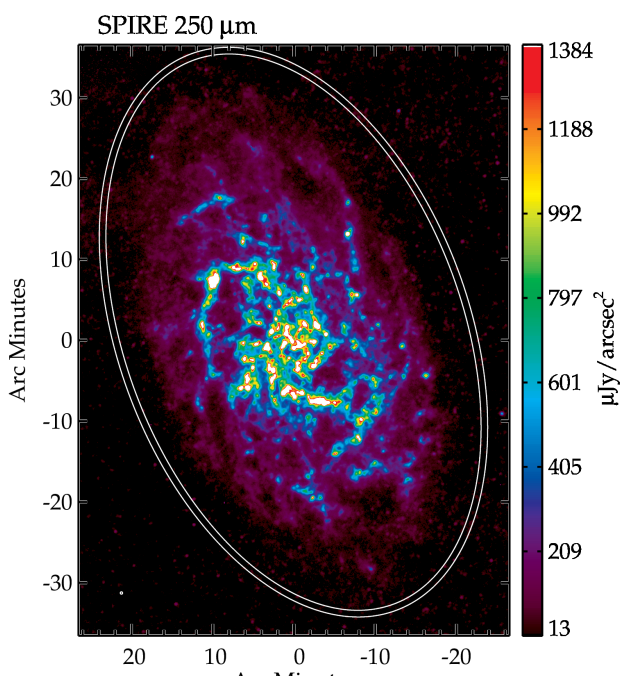

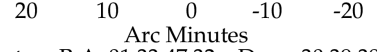

Center: R.A. 0133 47.32 Dec +30 3938.7

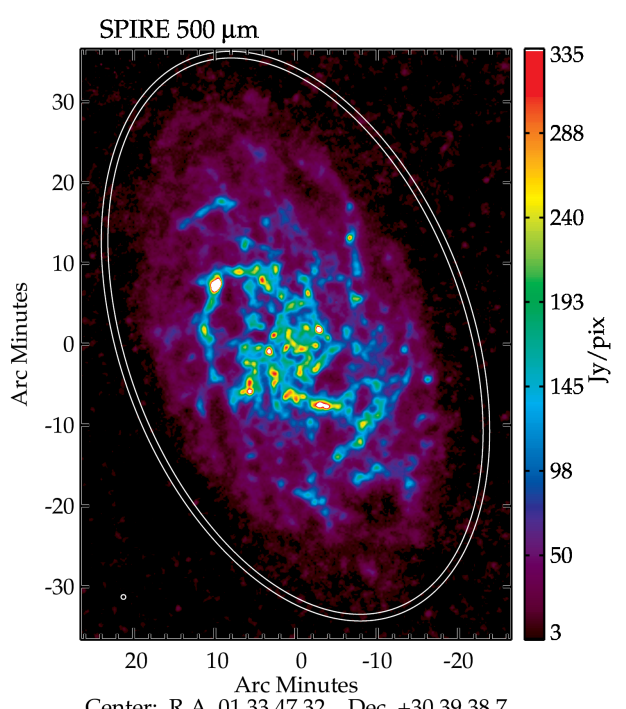

Center: R.A. 0133 47.32 Dec +30 3938.7

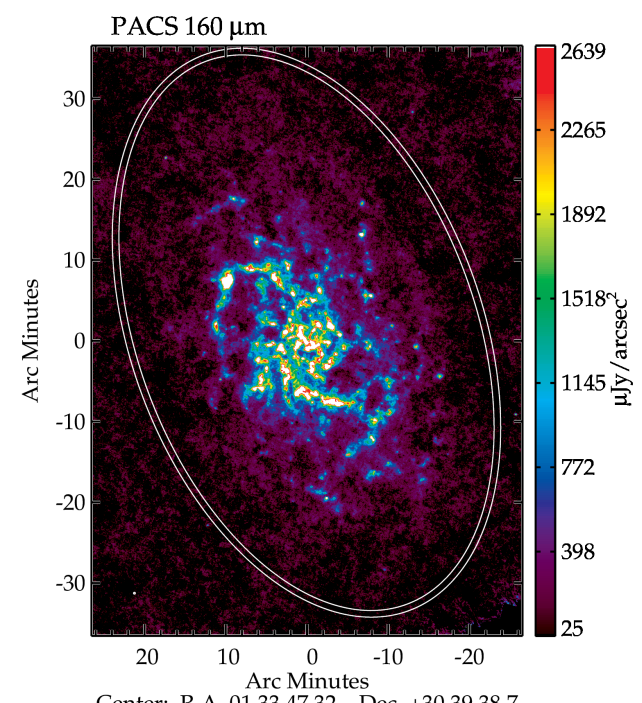

Center: R.A. 013347.32 Dec +303938.7

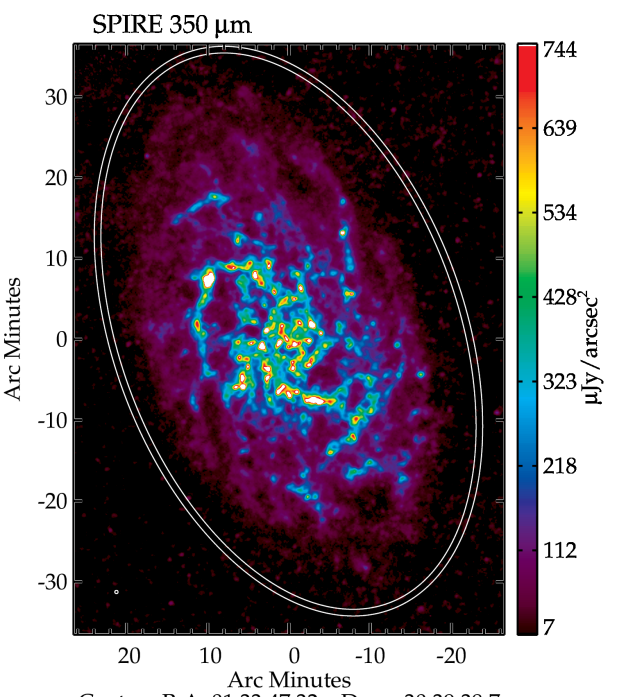

Center: R.A. 013347.32 Dec +30 3938.7

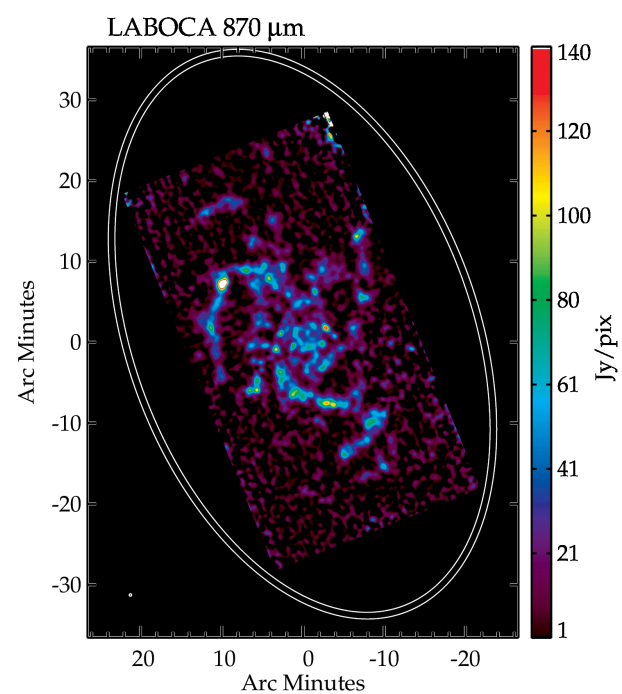

Center: R.A. 013347.32 Dec +30 3938.7

Fig. 1. continued. 
I. Hermelo et al.: Millimeter and submillimeter excess emission in M 33 revealed by Planck and LABOCA

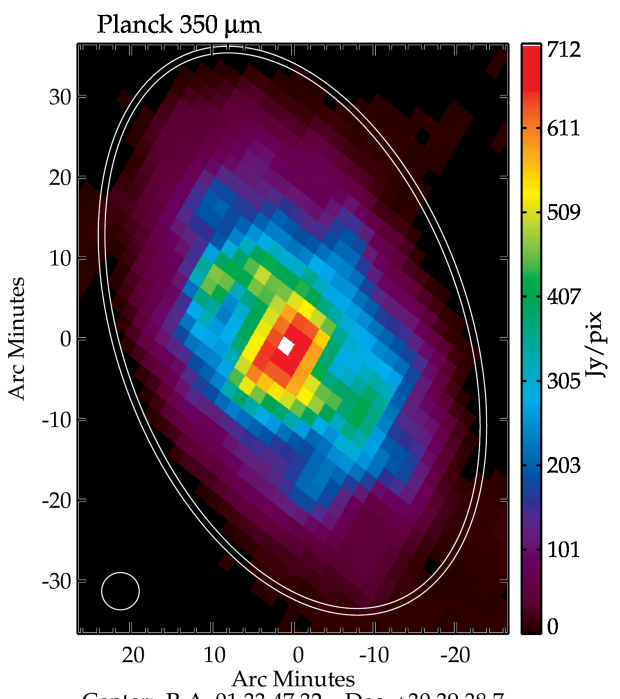

Center: R.A. 013347.32 Dec +303938.7

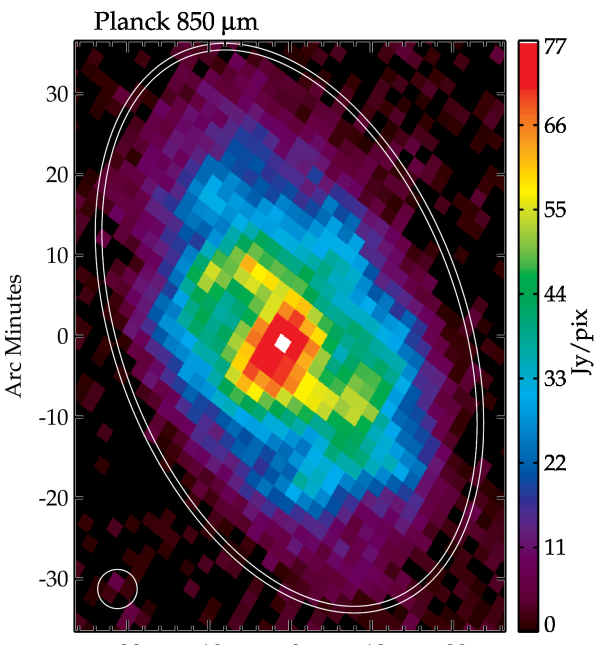

$\begin{array}{lllll}20 & 10 & 0 & -10 & -20\end{array}$

Center: R.A. 013347.32 Dec +303938.7

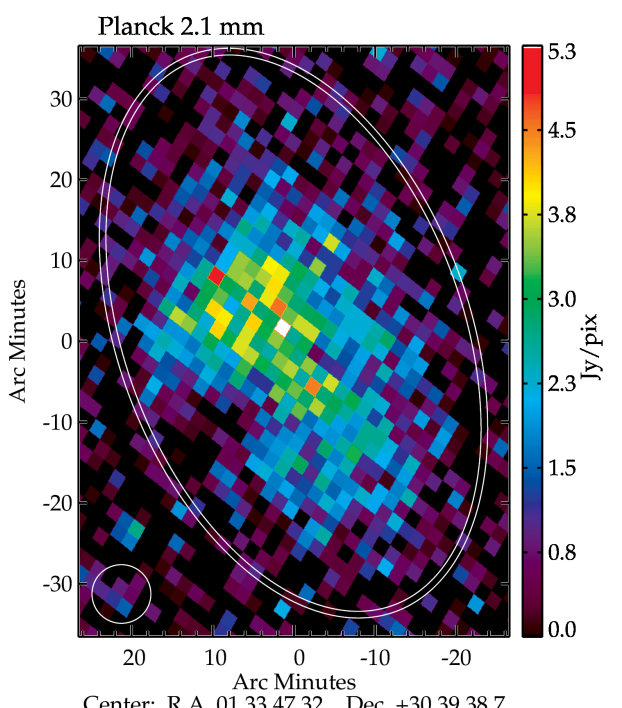

Center: R.A. 0133 Arc Minutes Dec +303938.7

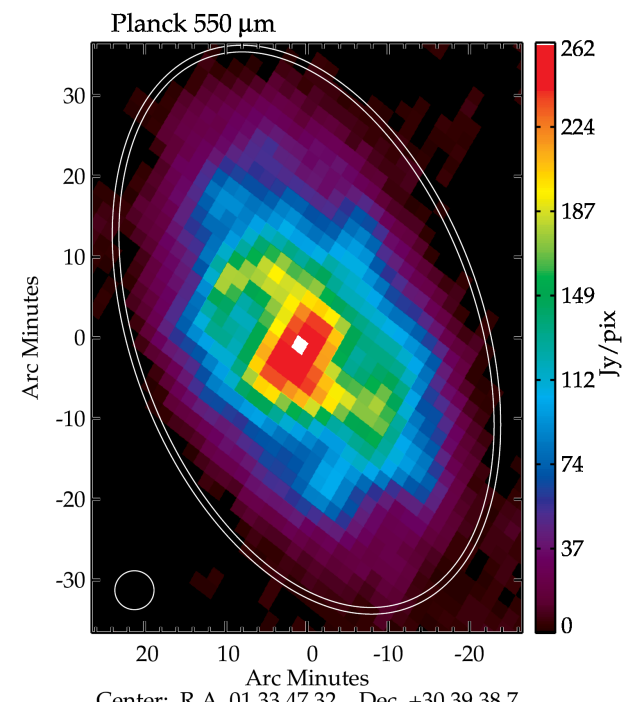

Center: R.A. 013347.32 Dec +303938.7

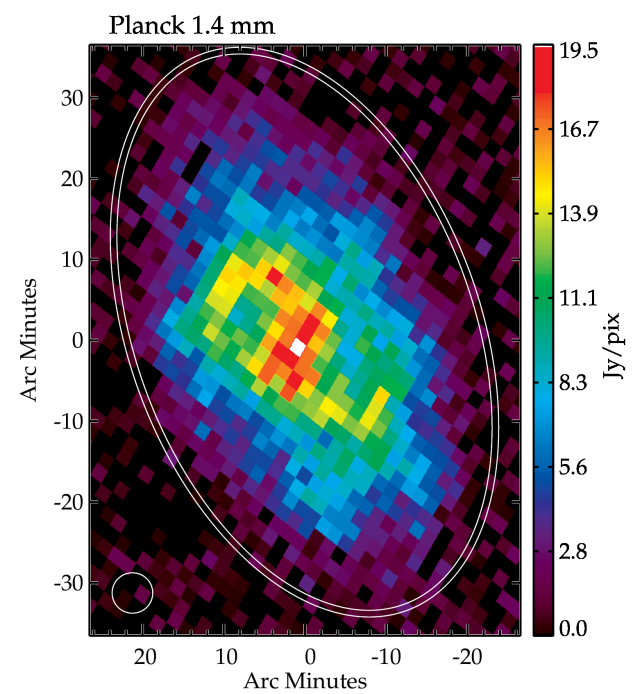

Center: R.A. 013347.32 Dec +303938.7

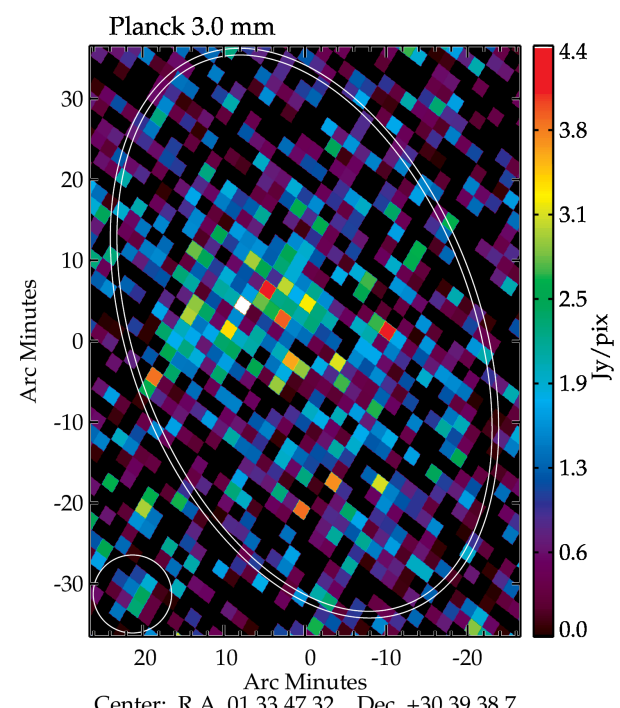

Fig. 1. continued. 

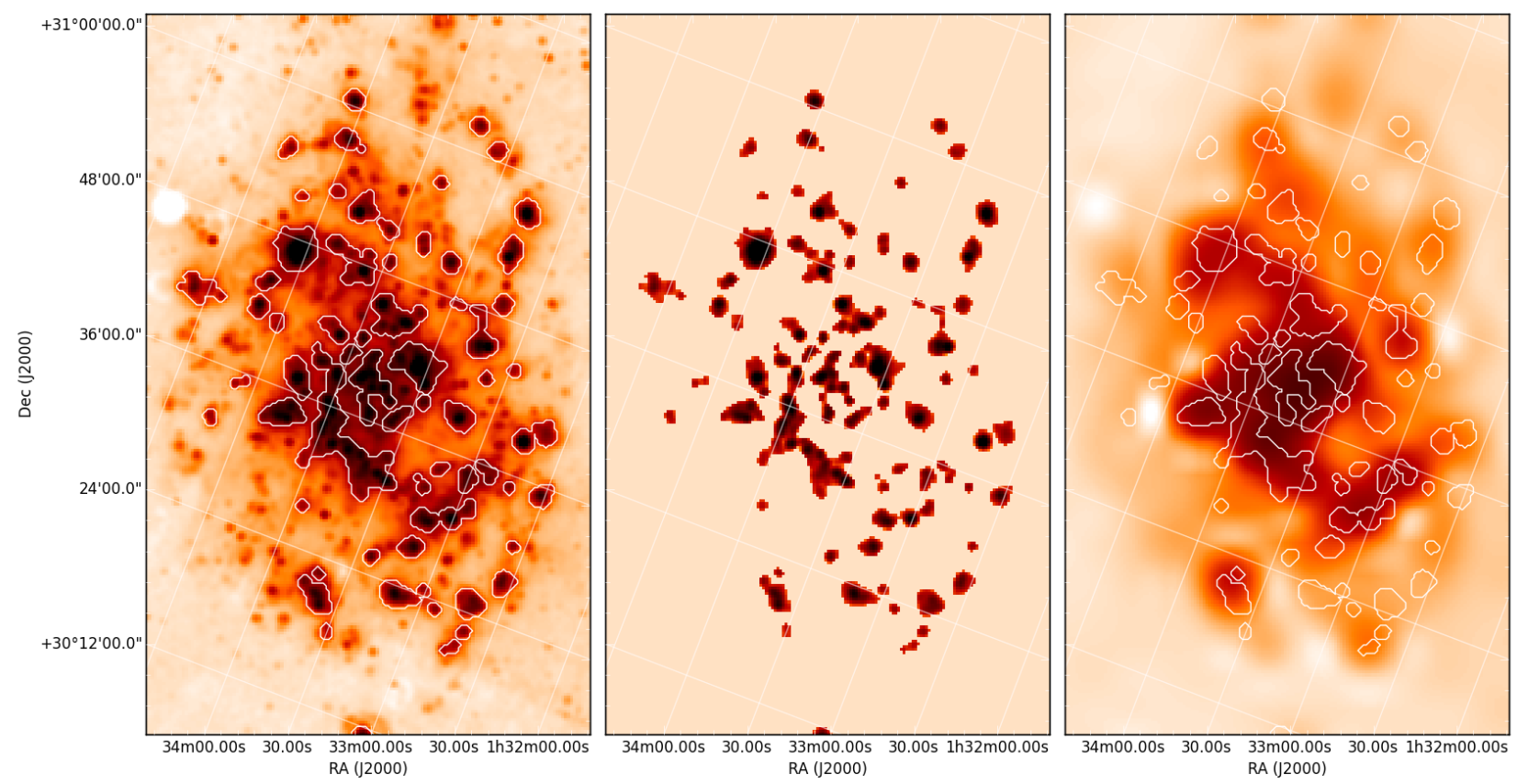

Fig. 2. Example of the component separation method for the case of the $\mathrm{H} \alpha$ emission. The left panel is the original $\mathrm{H} \alpha$ image smoothed to the resolution of the SPIRE $500 \mu \mathrm{m}$ band, the central panel shows the SF regions detected by SExtractor using a threshold of 40, and the right panel corresponds to the diffuse emission. The white contours are included to facilitate the comparison between panels and they correspond to the boundaries of the SF regions. The emission from the SF component represents $41.7 \%$ of the total $\mathrm{H} \alpha$ emission.

\subsection{Component separation}

In order to decompose M33 into its SF and diffuse components, we used the software SExtractor (Bertin \& Arnouts 1996). SExtractor explores a given image for knots of emission by searching for groups of connected pixels that exceed some threshold above the background in the surroundings of the region.

We ran SExtractor on the $\mathrm{H} \alpha$ image in order to separate the SF from the diffuse component. As the diffuse emission reaches a higher level in the inner part than in the outskirts of the galaxy, the threshold should be relative and should vary to reflect these local variations. The size of the background mesh should be large enough to avoid a strong contamination from the SF, but small enough to reproduce the local scale variations of the diffuse emission. We find that a mesh size of 12 pixels (with a pixel size of $14^{\prime \prime}$ which corresponds to $680 \mathrm{pc}$ ) yields an adequate separation between the detected SF objects and background map accounting for the diffuse emission. In practice, the decomposition between the SF and diffuse components can be summarized in two main steps. First, the diffuse emission map is computed by estimating the background (using a clipping to reject the bright SF regions) in each cell of a grid that covers the whole image. Second, SF regions are detected above a local threshold equal to 40 times the background noise map obtained in the previous step. The result of this separation method is shown in Fig. 2.

We tested different local thresholds between 5 and 100 times the background noise. We found that for values below 20 the SF regions detected are significantly contaminated by diffuse emission. On the other hand, values above 60 produce the rejection of many of the fainter SF regions. A good compromise that leads to an optimal separation of the two components was found for a threshold of 40 . We adopted this intermediate value and we discuss in Appendix $\mathrm{C}$ the impact of our choice.

To replicate the same separation in the other bands, we first derived a map of the diffuse emission in the same way as was done for the $\mathrm{H} \alpha$ map. We then obtained the difference between each original image and its diffuse emission map to remove the background/foreground diffuse emission from the whole map. Subsequently, we created a mask mirroring the detected SF objects in the $\mathrm{H} \alpha$ image and applied it to the full set of multiwavelength images to extract the exact same SF regions in all the bands. We used the $\mathrm{H} \alpha$ image to create the mask because this is the band that best represents the wavelength-dependent morphology of the SF regions. Finally, for each band, the diffuse emission map was obtained by subtracting the SF region map to the original map.

Once the SF and diffuse component were separated for each band we determined the flux densities by integrating over the corresponding maps (see Table 2). We used an aperture for the entire galaxy defined by a radius of $35^{\prime}(8.5 \mathrm{kpc})$ and an inclination of $57^{\circ}$ (see Fig. 1). This aperture is slightly larger than the one used by Kramer et al. (2010) in order to enclose the whole emission of the Planck bands, which have a much poorer resolution than the data used by these authors.

In those cases where the angular resolution did not allow the extraction of the emission from the SF regions (i.e., Planck data), the diffuse emission was estimated by subtracting the flux of the SF component predicted by the model of Groves et al. (2008) from the total flux of M33. Even though our estimates of the diffuse emission are well constrained by this method in most wavelengths, the lack of data points constraining the SED of the SF component between LABOCA $870 \mu \mathrm{m}$ and the free-free emission at $3.6 \mathrm{~cm}$ from Tabatabaei et al. (2007a) introduces some uncertainties. As a cautionary measure, we do not include the indirect data points in the fitting procedure.

\subsection{CO contamination}

The emission from the $\mathrm{CO}$ rotational transitions $\mathrm{CO}(1 \rightarrow 0)$ at $2.6 \mathrm{~mm}(115 \mathrm{GHz}), \mathrm{CO}(2 \rightarrow 1)$ at $1.3 \mathrm{~mm}(230 \mathrm{GHz})$, and 
Table 2. Flux densities of M 33 measured for the different bands.

\begin{tabular}{lcccc}
\hline \hline BAND & $S_{\mathrm{TOT}}^{\text {obs }}(\mathrm{Jy})$ & $S_{\mathrm{SF}}^{\text {obs }}(\mathrm{Jy})$ & $S_{\mathrm{DIFF}}^{\text {obs }}(\mathrm{Jy})$ & $S_{\mathrm{SF}}^{\text {obs }} / S_{\mathrm{TOT}}^{\text {obs }}(\%)$ \\
\hline GALEX FUV & $2.40 \pm 0.27$ & - & - & - \\
GALEX NUV & $3.52 \pm 0.39$ & - & - & - \\
Sloan $u$-band & $5.93 \pm 0.32$ & - & - & - \\
H $\alpha$ & $0.09 \pm 0.02$ & $0.04 \pm 0.007$ & - & 41.7 \\
IRAC $3.6 \mu \mathrm{m}$ & $23.7 \pm 1.26$ & - & - & - \\
IRAC $4.5 \mu \mathrm{m}$ & $15.3 \pm 0.82$ & - & - & - \\
MIPS $24 \mu \mathrm{m}$ & $47.6 \pm 3.05$ & $19.6 \pm 1.25$ & $29.2 \pm 2.37$ & 41.2 \\
PACS $70 \mu \mathrm{m}$ & $578 \pm 64.7$ & $223 \pm 25.0$ & $349 \pm 42.8$ & 38.7 \\
PACS $100 \mu \mathrm{m}$ & $1387 \pm 155$ & $360 \pm 40.3$ & $1026 \pm 125$ & 26.0 \\
PACS $160 \mu \mathrm{m}$ & $2162 \pm 445$ & $381 \pm 78.6$ & $1773 \pm 376$ & 17.6 \\
SPIRE $250 \mu \mathrm{m}$ & $1316 \pm 86.8$ & $197 \pm 13.1$ & $1111 \pm 92.0$ & 15.0 \\
Planck $350 \mu \mathrm{m}$ & $777 \pm 89.5$ & - & $684 \pm 78.8$ & 12.0 \\
SPIRE $350 \mu \mathrm{m}$ & $718 \pm 47.4$ & $90.5 \pm 5.97$ & $632 \pm 52.3$ & 12.6 \\
SPIRE $500 \mu \mathrm{m}$ & $319 \pm 21.0$ & $37.5 \pm 2.47$ & $279 \pm 23.1$ & 11.7 \\
Planck $550 \mu \mathrm{m}$ & $305 \pm 35.1$ & - & $279 \pm 32.2$ & 8.3 \\
Planck $850 \mu \mathrm{m}$ & $91.9 \pm 6.91$ & - & $85.7 \pm 6.45$ & 6.8 \\
LABOCA $870 \mu \mathrm{m}$ & - & $10.0 \pm 1.12$ & - & - \\
Planck $1.4 \mathrm{~mm}$ & $21.7 \pm 1.95$ & - & $20.3 \pm 1.83$ & 6.5 \\
Planck $2.1 \mathrm{~mm}$ & $4.27 \pm 0.57$ & - & $3.66 \pm 0.49$ & 14.2 \\
Planck $3.0 \mathrm{~mm}$ & $1.86 \pm 0.19$ & - & $1.41 \pm 0.14$ & 24.2 \\
Planck $10 \mathrm{~mm}$ & $0.40 \pm 0.09$ & - & - & - \\
S 36 mm & $0.44 \pm 0.05$ & $0.44 \pm 0.05$ & - & 100.0 \\
\hline
\end{tabular}

Notes. The total emission ( $\left.S_{\mathrm{TOT}}^{\mathrm{obs}}\right)$ is shown in Col. 2. Columns 3 and 4 correspond to the emission from the $\mathrm{SF}\left(S_{\mathrm{SF}}^{\mathrm{obs}}\right)$ and the diffuse $\left(S_{\mathrm{TOT}}^{\mathrm{obs}}\right)$ component. Column 5 indicates the percentage of the total flux emitted by the SF component.

$\mathrm{CO}(3 \rightarrow 2)$ at $870 \mu \mathrm{m}(345 \mathrm{GHz})$ fall into the bandpasses of the Planck $3.0 \mathrm{~mm}, 1.4 \mathrm{~mm}$, and $850 \mu \mathrm{m}$ filters, respectively (Planck Collaboration XIII 2014). In addition, the LABOCA $870 \mu \mathrm{m}$ filter is also affected by the $\mathrm{CO}(3 \rightarrow 2)$ line. In order to correct the measured continuum fluxes for the contribution of these major gas cooling lines, we made use of $\mathrm{CO}(2 \rightarrow 1)$ observations (Druard et al. 2014) obtained with the HEterodyne Receiver Array (HERA, Schuster et al. 2004) mounted on the IRAM 30 m telescope on Pico Veleta (Spain).

Druard et al. (2014) mapped the inner $\sim 7 \mathrm{kpc}$ of M 33 with an angular resolution of $12^{\prime \prime}$. These authors reported a total $\mathrm{CO}(2 \rightarrow 1)$ luminosity of $2.8 \times 10^{7} \mathrm{~K} \mathrm{~km} \mathrm{~s}^{-1} \mathrm{pc}^{2}$, which corresponds to a flux of $6.5 \times 10^{4} \mathrm{Jy} \mathrm{km} \mathrm{s}^{-1}$. For the PLANCK $1.4 \mathrm{~mm}$ filter, which has a width of $64.5 \mathrm{GHz}\left(87433 \mathrm{~km} \mathrm{~s}^{-1}\right)$ and a transmission close to $100 \%$ at the frequency of the $\mathrm{CO}(2 \rightarrow 1)$ line (see Fig. 1 in Planck Collaboration XIII 2014), we measured a flux density of $17.4 \mathrm{Jy}$. Using these values we estimate that $4.3 \%$ of the flux density of M 33 measured with this filter comes from the $\mathrm{CO}(2 \rightarrow 1)$ line emission.

The $\operatorname{CO}(1 \rightarrow 0)$ line emission was also observed with the IRAM $30 \mathrm{~m}$ telescope in a radial cut along the major axis of M 33 (Braine et al. 2010). Comparing these data with their map, Druard et al. (2014) found a constant ratio $I_{\mathrm{CO}(1 \rightarrow 0)} / I_{\mathrm{CO}(2 \rightarrow 1)}$ of 1.25 in the overlapping area. Assuming that this ratio can be extrapolated to the whole disk of M 33, we can estimate the flux of $\mathrm{CO}(1 \rightarrow 0)$ as

$S_{\mathrm{CO}(1 \rightarrow 0)}=1.25 \times\left(\frac{115}{230}\right)^{2} S_{\mathrm{CO}(2 \rightarrow 1)}=2.0 \times 10^{4} \mathrm{Jy} \mathrm{km} \mathrm{s}^{-1}$.

We measured a flux density of $1.56 \mathrm{Jy}$ with the Planck $3.0 \mathrm{~mm}$ filter, which has a width of $32.9 \mathrm{GHz}\left(97762 \mathrm{~km} \mathrm{~s}^{-1}\right)$ and a transmission of about $60 \%$ at the frequency of the line. Therefore, we estimate that the $\mathrm{CO}(1 \rightarrow 0)$ emission is responsible for $7.9 \%$ of the flux measured with the Planck $3.0 \mathrm{~mm}$ band.

Finally, the contamination due to the emission from the $\mathrm{CO}(3 \rightarrow 2)$ line was estimated assuming a ratio $I_{\mathrm{CO}(3 \rightarrow 2)} / I_{\mathrm{CO}(2 \rightarrow 1)} \sim 1$, i.e.,

$S_{\mathrm{CO}(3 \rightarrow 2)} \sim\left(\frac{345}{230}\right)^{2} S_{\mathrm{CO}(2 \rightarrow 1)}=14.6 \times 10^{4} \mathrm{Jy} \mathrm{km} \mathrm{s}^{-1}$.

With the Planck $850 \mu \mathrm{m}$ filter we measured a flux density of $74.3 \mathrm{Jy}$. Using a width of $101.4 \mathrm{GHz}\left(85032 \mathrm{~km} \mathrm{~s}^{-1}\right)$ and a transmission $\sim 100 \%$, we found that the contribution from the $\mathrm{CO}(3 \rightarrow 2)$ line to the Planck $850 \mu \mathrm{m}$ is $\sim 2.3 \%$. In the case of the LABOCA $870 \mu \mathrm{m}$ filter, we measured a flux density of $37.1 \mathrm{Jy}$. For a width of $59.8 \mathrm{GHz}\left(52026 \mathrm{~km} \mathrm{~s}^{-1}\right)$ and a transmission $\sim 100 \%$, we found that the contribution from the $\mathrm{CO}(3 \rightarrow 2)$ line to this filter is $(\sim 7.6 \%)$.

\subsection{Synchrotron emission}

Unlike the free-free emission coming from the H II regions, the modeling applied in this work does not take into account the contribution of the synchrotron emission. For this reason, the fluxes measured with Planck need to be decontaminated from this component. Following the analysis of Tabatabaei et al. (2007a) we extrapolated the synchrotron emission from $3.6 \mathrm{~cm}$ to the Planck filters assuming a non-thermal spectral index of 0.72 (see Sect. 2.8). We found non-thermal contributions of $30 \%$ and $5 \%$ respectively for the Planck $10 \mathrm{~mm}$ and $3.0 \mathrm{~mm}$ bands, while for the other bands this contribution was found to be negligible. 


\subsection{Error of flux measurements}

In our error analysis we took two types of error into account: i) calibration errors, $\Delta_{\text {cal }}$, using the values presented in Table 1; and ii) measurement errors due to background fluctuations, $\Delta_{\text {back }}$.

The error due to the background fluctuations was calculated by assuming that each pixel within the aperture has an error given by the standard deviation of the background noise, $\sigma_{\text {back }}$. In addition, we have to take into account the error of the background that was subtracted within an aperture of $N_{\text {apert }}$ pixels. This error is $\sigma_{\text {back }} N_{\text {apert }} / \sqrt{N_{\text {back }}}$, where $N_{\text {back }}$ is the number of pixels used to compute the level of background. This gives (see also Dale et al. 2012) a total error for the background subtracted flux of

$\Delta_{\text {back }}=\sigma_{\text {back }} \sqrt{N_{\text {apert }}+\frac{N_{\text {apert }}^{2}}{N_{\text {back }}}}$.

The final error for the flux is the quadratic sum of $\Delta_{\text {cal }}$ and $\Delta_{\text {back }}$. In the case of the Planck data, $\Delta_{\text {back }}$ also includes the error due to the CMB subtraction.

\section{Models for the dust emission}

We analyzed the full UV to radio SED of the different emission components of the galaxy using the radiation transfer model of Popescu et al. (2011), which self-consistently treats the dust emission from the SF and the diffuse components, considering the illumination of the diffuse dust both by the distributed stellar populations and by the escaping light from the H II regions. While maintaining consistency within the framework of the Popescu et al. (2011) model, we used the model of Groves et al. (2008) to provide a detailed description of the dust emission from the SF component. The methodology used in this work is similar to the one applied to the low-metallicity, dwarf galaxy NGC 4214 in Hermelo et al. (2013). For a detailed description of the models we refer the reader to Groves et al. (2008) and Popescu et al. (2011). For convenience, in the following sections we present a brief description of the physics and parameters of the models.

\subsection{The Popescu et al. (2011) model}

Popescu et al. (2011) present a self-consistent model based on full radiative transfer calculations of the propagation of starlight in disk galaxies. These authors adopt the dust properties from Weingartner \& Draine (2001) and Draine \& Li (2007), incorporating a mixture of graphite, silicate, and PAH molecules. To approximate the large-scale geometry of the galaxy (see Fig. 1 in Popescu et al. 2011), they use two separate components:

- an old component consisting of an old stellar disk, an old stellar bulge, and a thick disk of dust;

- a young component consisting of a young stellar disk and a thin disk of dust.

The spectral energy distribution of both old and young stellar components are shown in Table E. 2 and in Fig. 8 of Popescu et al. (2011). These authors only considered the opticalIR radiation for the old stellar population and neglected any contribution in the UV. The spectral distribution covers the wavelengths from $4430 \AA$ to $50000 \AA$. The young stellar population is defined as an exponentially declining $S F R$ with a time constant of $5 \mathrm{Gyr}$, solar metallicity, and Salpeter IMF with an upper mas cut-off of $100 M_{\odot}$. The spectral distribution covers from $912 \AA$ to $50000 \AA$.

Apart from the diffuse component, the model includes a clumpy component, consisting of the parent molecular clouds of young ( $\leq 10 \mathrm{Myr})$ and massive stars. This component will be modeled here in a different way than in Popescu et al. (2011) (see Sect. 4.2) as for M 33 it is possible to separate the emission from the SF regions and the diffuse dust.

The input parameters of the Popescu et al. (2011) model are

- the total central face-on $B$-band opacity, $\tau_{B}^{\mathrm{f}}$;

- the star formation rate, $S F R$;

- the clumpiness factor $F$, which is linked to the fraction of photons that escape $\left(f_{\text {esc }}\right)$ from the SF regions into the diffuse medium $\left(F=1-f_{\text {esc }}\right)$;

- the normalized luminosity of the old stellar disk, old;

- the bulge-to-disk ratio, $B / D$;

- the radial scale length of the old stellar disk, $h_{\mathrm{s}}$. All other spatial scales in the galaxy related to the different component in the model have a constant ratio with $h_{\mathrm{s}}$ (see Table E.1 in Popescu et al. 2011);

- the inclination angle of the galaxy, $i$.

From the primary parameters $S F R$ and $F$, Popescu et al. (2011) define the SFR powering the diffuse emission, $S F R^{\prime}$, as follows (Eq. (45) in Popescu et al. 2011):

$S F R^{\prime}=S F R \times(1-F)$.

The library of diffuse SEDs of Popescu et al. (2011) contains results for a four-dimensional parameter space spanned by $\tau_{B}^{\mathrm{f}}$, $S F R^{\prime}$, old, and $B / D$.

The diffuse component is calculated as an extrinsic quantity corresponding to a reference size (corresponding to a reference scalelength). To scale the intensity of the radiation field heating the diffuse dust, the parameters $S F R^{\prime}$ and old must be scaled to the reference size by comparing the scalelengths (Eq. (D.3) in Popescu et al. 2011)

$S F R^{\text {mod }}=S F R^{\prime} \times\left(\frac{h_{\mathrm{ref}}}{h_{\mathrm{s}}}\right)^{2}$

and

$o l d^{\mathrm{mod}}=$ old $\times\left(\frac{h_{\mathrm{ref}}}{h_{\mathrm{s}}}\right)^{2}$,

where $h_{\text {ref }}=5670 \mathrm{pc}$ is the reference $B$-band scalelength, $h_{\mathrm{s}}$ is the $B$-band scalelength of the galaxy under study, and $S F R^{\text {mod }}$ and old ${ }^{\text {mod }}$ are internal parameters that allow us to interface with the library of models. An additional scaling is required to set the flux levels of the SEDs from the library, $S_{v}^{\text {d,mod }}$, to the SED that represents our galaxy, $S_{v}^{\mathrm{d}}$,

$S_{v}^{\mathrm{d}}=\left(\frac{h_{\mathrm{s}}}{h_{\mathrm{ref}}}\right)^{2} \times S_{v}^{\mathrm{d}, \mathrm{mod}}$,

where $S_{v}^{\text {d,mod }}$ is determined by the parameters $\tau_{B}^{\mathrm{f}}, S F R^{\mathrm{mod}}$, old $^{\text {mod }}$, and $B / D$ (Popescu et al. 2011, Eq. (D.2)). We note that in these models the absolute flux level of the predicted dust SED is fixed by the input parameters.

In the model of Popescu et al. (2011), the stellar radiation field leaving the galaxy, $S_{v}^{\mathrm{s}}$, can be calculated as

$S_{v}^{\mathrm{s}}=S_{v}^{\mathrm{s}, \mathrm{int}} \times 10^{-\Delta m_{\lambda} / 2.5}$ 
where $S_{v}^{\text {s,int }}$ is the intrinsic stellar radiation field and $\Delta m_{\lambda}$ is the composite attenuation. In the case of the UV and optical emission coming from the young stellar population, $\Delta m_{\lambda}$ can be written as

$\Delta m_{\lambda} \simeq-2.5 \log \left(1-F f_{\lambda}\right)+\Delta m_{\lambda}^{\text {tdisk }}$.

The first part of Eq. (10) takes into account the attenuation in the SF component, while $\Delta m_{\lambda}^{\text {tdisk }}$ is responsible for the attenuation in the diffuse component. The wavelength dependence of the escape fraction, $f_{\lambda}$, is tabulated in Tuffs et al. (2004, Table A.1). Therefore, by combining Eqs. (9) and (10), it is possible to calculate the radiation field associated with the young stellar population that escapes the galaxy $\left(S_{v}^{\text {s,young }}\right)$. The emission of the old stars that leaves the galaxy $\left(S_{v}^{\text {s,old }}\right)$ is calculated in a similar way, but in this case the composite attenuation is $\Delta m_{\lambda}=\Delta m_{\lambda}^{\text {tdisk }}$, since this radiation field is only attenuated by the diffuse ISM.

It is noteworthy that in the Popescu et al. (2011) model the old stellar population emits exclusively in the optical-IR wavelength range (see their Table E.2), i.e., the emission corresponds to the radiation field emitted by a remarkably old stellar population. Thus, what Popescu et al. (2011) calls "old" stellar population does not correspond to the definition of "old" stellar population usually found in the literature (see Fig. 1, panel Scd, in Rowan-Robinson et al. 2008 for an example of a moderate old stellar population with a SED extending into the optical-UV range). As a consequence, in the Popescu et al. (2011) model the old stellar population has little relevance for the dust heating.

\subsection{The Groves et al. (2008) model}

In Popescu et al. (2011) the clumpy component corresponding to the $\mathrm{SF}$ regions is modeled with an average template representing the emission of all SF regions. Although this is a good approach for unresolved galaxies, the high spatial resolution of our data allowed us to apply a detailed modeling of the SF component.

The Groves et al. (2008) model describes the luminosity evolution of a star cluster of mass $M_{\mathrm{cl}}$, and incorporates the expansion of the HII region and PDR due to the mechanical energy input of stars and supernovae. The dust emission from the HII region and the surrounding PDR is calculated from radiation transfer calculations. The hydrogen column density of the PDR is fixed to the value $N_{\mathrm{HI}}^{\mathrm{PDR}}=10^{22} \mathrm{~cm}^{-2}$. The Groves et al. (2008) model assumes a standard mixture of dust consisting of graphite, silicate, and PAHs, with standard grain properties from Laor \& Draine (1993), Li \& Draine (2001), and Weingartner \& Draine (2001). The input parameters of the model are

- the metallicity of the star cluster, $Z$, in units of the solar metallicity $Z_{\odot}$ (Asplund et al. 2005);

- the age of the star cluster, $\mathcal{T}$, in Myr;

- the ambient pressure, expressed as

$$
p_{0}=\log \left(\frac{n_{0} T_{0}}{\mathrm{~cm}^{-3} \mathrm{~K}}\right) \text {, }
$$

where $n_{0}$ and $T_{0}$ are respectively the number density and the temperature of the surrounding ISM;

- the compactness parameter, $C$, which parametrizes the heating capacity of the star cluster and depends on $M_{\mathrm{cl}}$ and $p_{0}$ as

$$
C=\frac{3}{5} \log \left(\frac{M_{\mathrm{cl}}}{M_{\odot}}\right)+\frac{2}{5} p_{0} .
$$

The $C$ parameter determines the dust grain temperature distribution;
- the covering factor, $f_{\text {cov }}$, which represents the fraction of the surface of the HII region covered by the PDR. We note that $f_{\text {cov }}$ is the same parameter as the $F$ factor in the Popescu et al. (2011) model. Hereafter the abbreviation $f_{\text {cov }}$ is used to refer to both parameters.

\subsection{Observational constraints on the input parameters}

The large amount of ancillary data for M33, as well as the results of previous studies from the literature, allowed us to constrain part of the input parameters for both models.

For the model of Popescu et al. (2011) we were able to place the following constraints:

- Bulge-to-disk ratio: using deep $H$-band $(1.65 \mu \mathrm{m})$ observations of M33, Minniti et al. (1993) found an excess of emission in the innermost $2^{\prime}$ that they identified with a small bulge. Regan \& Vogel (1994) claimed that if a compact bulge is present, it does not make a large contribution to the spheroidal light. More recently, Corbelli \& Walterbos (2007) analyzed gas and stellar radial velocities in the innermost $0.5 \mathrm{kpc}$ of M33. Their study showed that the central part of the galaxy does not exhibit kinematic signature of a significant bulge. Since there is no convincing evidence for a bulge in M 33, here we opted for a bulge-to-disk ratio $(B / D)$ equal to 0 .

- Inclination angle: we assumed an inclination angle of $57^{\circ}$ based on $21 \mathrm{~cm}$ line observations of M33 with the Westerbork Synthesis Radio Telescope (Deul \& van der Hulst 1987).

- B-band scalelength: we obtained the radial stellar scale length in the $B$-band, $h_{\mathrm{s}}$, using the Sloan $g$-band image. The fit of an exponential function to the surface brightness profile gives a value of $h_{\mathrm{s}}=2513 \pm 80 \mathrm{pc}$ (see Fig. 3). This value agrees with the scalelength for young star formation tracers such as FUV, NUV, and $\mathrm{H} \alpha$ (Verley et al. 2009).

- Dust scalelength: in the model of Popescu et al. (2011) the scale of the different components are normalized to the $B$-band scalelength of the disk, $h_{\mathrm{s}}$. In particular, the Popescu et al. (2011) model assumes that the scalelength of the thick disk of dust $\left(h_{\mathrm{d}}\right)$ is a factor of 1.4 larger than $h_{\mathrm{s}}$. To compare the actual geometry of M 33 with the model assumptions we calculated the scalelength traced by the SPIRE $500 \mu \mathrm{m}$ emission. Shorter wavelengths are more affected by the dust temperature distribution, while Planck data at longer wavelengths lack the angular resolution necessary to extract isophotal fluxes. For SPIRE $500 \mu \mathrm{m}$ we found a scalelength of $3200 \pm 100 \mathrm{pc}$ (see Fig. 4). If we approximate $h_{\mathrm{d}}$ with this value, we obtain $h_{\mathrm{d}} / h_{\mathrm{s}}=1.27 \pm 0.08$, close to the value 1.4 assumed in the model.

- old: we derived this parameter as 0.05 by assuming that the IRAC $3.6 \mu \mathrm{m}$ and $4.5 \mu \mathrm{m}$ bands mainly trace the emission from the old stellar population (Bendo et al. 2015). Then we integrated the luminosity of M 33 under these two bands and derived old as the ratio of this luminosity and the integrated luminosity of the model galaxy for the old stellar component (Popescu et al. 2011, Table E2).

In the case of the Groves et al. (2008) model, we established the following constraints:

- Metallicity: Magrini et al. (2007) have studied the radial oxygen abundance gradient in M33 using a list of 83 H II regions with [O III] $\lambda 4363$ detections. These authors find that $12+\log [\mathrm{O} / \mathrm{H}]$ ranges from $\sim 8.5$ to 8.1 (see their Fig. 3). For 


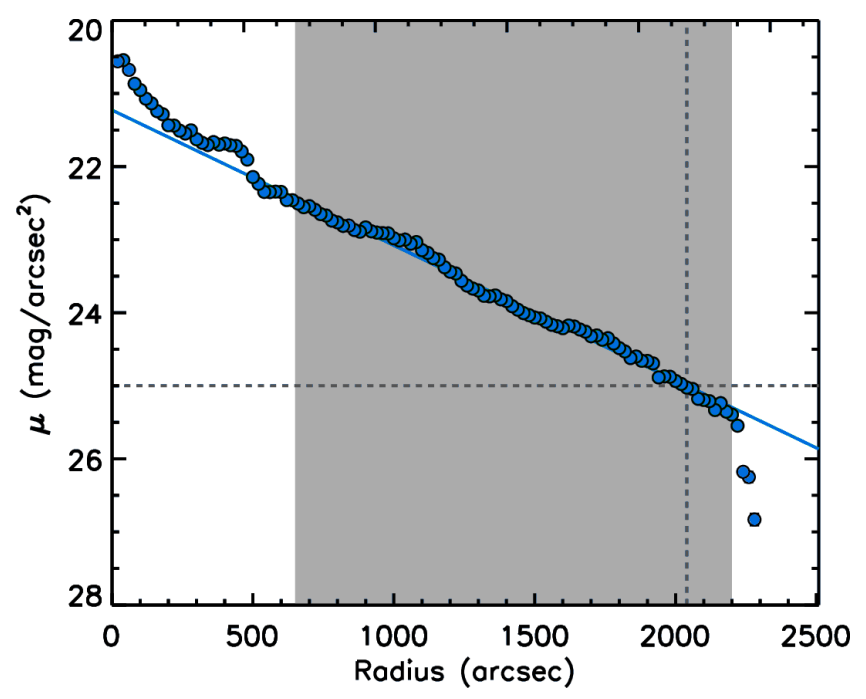

Fig. 3. $B$-band surface brightness of the stellar disk of M 33 plotted against deprojected radius. The background subtracted Sloan $g$-band was used to obtain the average value of circular apertures in the outer parts of M33 disk where previously foreground stars and H II regions were masked. The fit (solid line) was achieved using the isophotes in the radial range marked by the gray-shaded area. We obtain a final scalelength of $2513 \pm 80 \mathrm{pc}$. The horizontal and vertical dashed gray lines indicate the $25 \mathrm{mag} / \mathrm{arcsecond}^{2}$ isophote. The error bars are smaller than the data points.

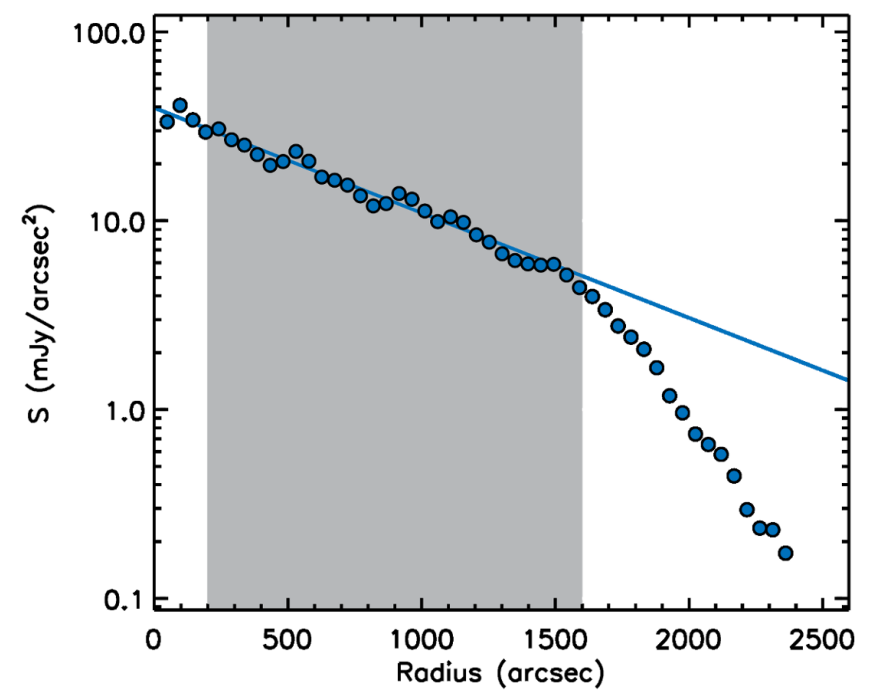

Fig. 4. SPIRE $500 \mu \mathrm{m}$ surface brightness of M 33 plotted against deprojected radius. The fit (solid line) was achieved using the isophotes in the radial range marked by the grey-shaded area. The error bars are smaller than the data points.

the solar metallicity presented in Asplund et al. (2005) these limits correspond to values of $Z_{\odot}$ from $\sim 0.7$ to 0.3 . To cover this range we constrained the metallicity to the discrete values $1.0,0.4$, and $0.2 Z_{\odot}$ allowed by the Groves et al. (2008) model.

- Age: a small sample of young stellar clusters has been studied by Grossi et al. (2010) and Fan \& de Grijs (2014), who find that most of the star clusters have ages of $~ 3 \mathrm{Myr}$. A larger sample has been studied by Sharma et al. (2011), who find representative values of about $5 \mathrm{Myr}$ in the inner part of the disk (galactocentric radii $\lesssim 4 \mathrm{kpc}$ ) and about $7 \mathrm{Myr}$ for star clusters within the outer disk (from 4 to $7 \mathrm{kpc}$ ). Therefore, we restricted the age to the range from 3 to $7 \mathrm{Myr}$.
Table 3. List of the parameters used in our modeling.

\begin{tabular}{lcccc}
\hline \hline Parameter & Abbr. & Units & Range & Value \\
\hline Metallicity & $Z$ & $Z_{\odot}$ & $0.2-1.0$ & $0.2_{\vee 0.4}^{\wedge 0.2}$ \\
Age & $\mathcal{T}$ & $\mathrm{Myr}$ & $3.0-7.0$ & $4.0_{\vee 4.0}^{\wedge 4.5}$ \\
Compactness & $C$ & - & Free & $4.0_{\vee 4.0}^{\wedge 4.0}$ \\
Ambient pressure & $p_{0}$ & - & Free & $8_{\vee 8}^{\wedge}$ \\
Covering factor & $f_{\text {cov }}$ & $\%$ & Free & $0.46_{\vee 0.74}^{\wedge 0.36}$ \\
Opacity & $\tau_{B}^{\mathrm{f}}$ & - & Free & $2.2_{\vee 1.6}^{\wedge 2 .}$ \\
Star formation rate & $S F R^{\prime}$ & $M_{\odot} \mathrm{yr}^{-1}$ & Free & $0.25_{\vee 0.20}^{\wedge 0.29}$ \\
Old stellar luminosity & old & - & Fixed & 0.05 \\
Bulge-to-disk ratio & $B / D$ & - & Fixed & 0.0 \\
Inclination angle & $i$ & $\mathrm{deg}$ & Fixed & 57 \\
$B$-band scalelength & $h_{\mathrm{s}}$ & $\mathrm{pc}$ & Fixed & 2513 \\
\hline
\end{tabular}

Notes. The names of the parameters are shown in Col. 1, the abbreviations used in the text in Col. 2, the units in Col. 3, and the observational constraints used to obtain the best-fitting solutions in Col. 4. The median, minimum, and maximum values derived from the fitting procedure are specified in Col. 5.

- Compactness and ambient pressure: unfortunately, we could not constrain either $C$ or $p_{0}$, so we left both parameters free in the fitting procedure and checked later whether they were within the expected range of values derived from Eq. (12) and the mass range derived by Sharma et al. (2011) for the star clusters in the inner part of M33 (from $10^{2} M_{\odot}$ to $\left.10^{5} M_{\odot}\right)$.

The remaining parameters were left free in the fitting procedure.

\subsection{Fitting procedure}

In order to find the best-fitting model and its uncertainty for both the SF and the diffuse components we used the following procedure. For each component we generated a total of 100 slightly different data sets by allowing the observed fluxes to randomly vary following a Gaussian distribution within the error bars. For each of these sets we found the best-fitting model using a $\chi^{2}$ minimization technique. To build the best-fitting solution (i.e., the most probable SED and its range of uncertainty) we extracted the median, the minimum, and the maximum SEDs from the set of 100 best-fitting models. To express the range of values obtained through the iteration process we will use the nomenclature $\mathrm{Xmed} \underset{\vee \mathrm{Xmin}}{\wedge \mathrm{Xmax}}$ to report median (Xmed), maximum (Xmax), and minimum (Xmin) values.

\section{Results}

\subsection{Best fit for the SF component}

The best-fitting solution for the SF component was obtained by exploring the parameter space of the Groves et al. (2008) model with the constraints presented in Table 3 (see Sect. 4.3 for details). For each of the 100 iterations, a total of 6885 different parameter combinations were tested to find the minimum $\chi^{2}$. We note that Planck data could not be used to fit the SF component owing to their lack of angular resolution. The best-fitting model is shown in Fig. 5, and the range of values found for the parameters is presented in Table 3 . The reduced $\chi^{2}$ is $4.69_{\vee 0.73}^{\wedge}$. We found that the metallicity of the best-fitting solution is $0.2 \wedge 0.4$, at the low end of the values reported by Magrini et al. (2007). 


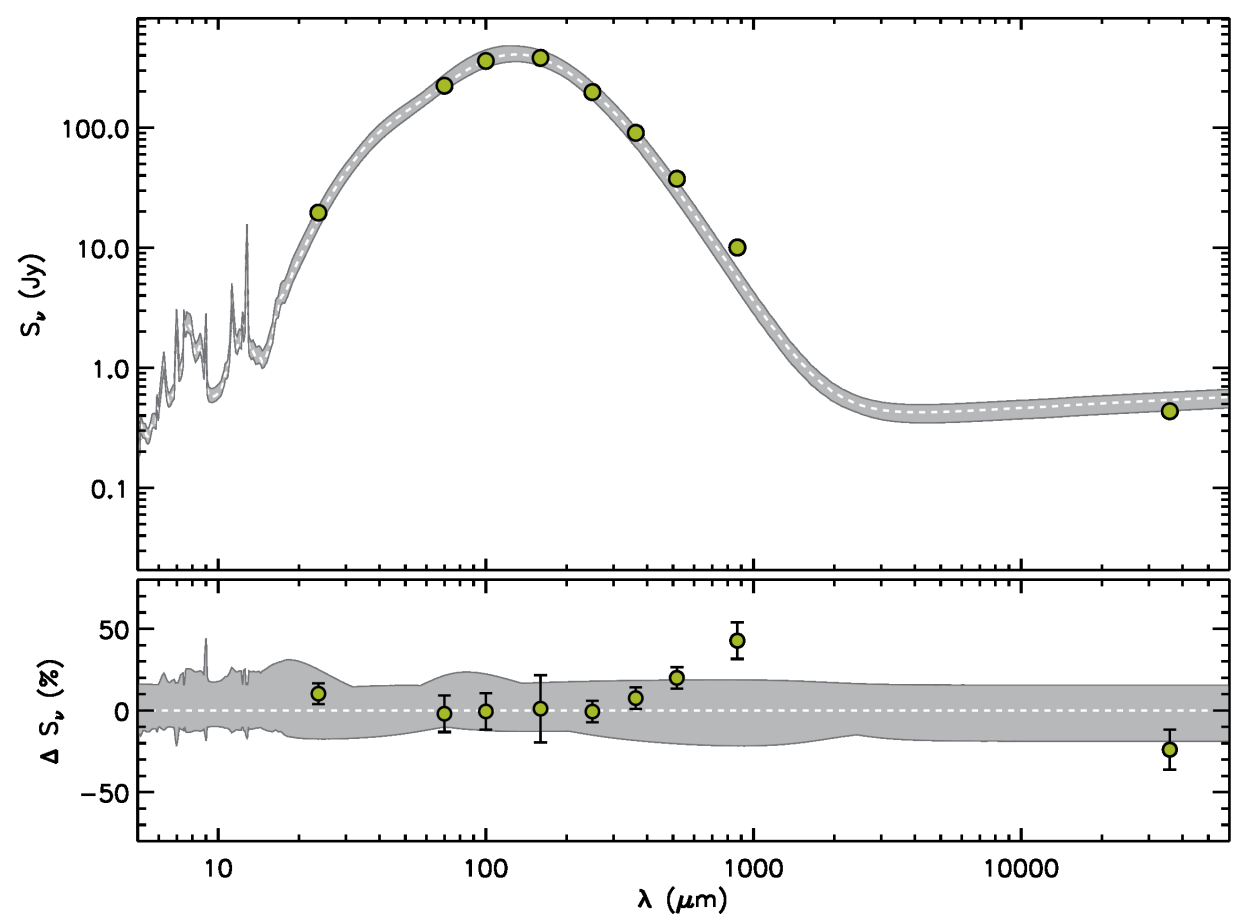

Fig. 5. Top: results of the fitting procedure for the SF component of M 33 . The green filled circles are the observed fluxes for the SF component presented in Table 2 . The best-fitting model is represented by the white dashed line, while its uncertainty is defined by the gray shaded area. For clarity, errors are only shown in the bottom panel. Bottom: residuals of the fitting procedure. The green filled circles correspond to the percentage difference of the observed fluxes and the best-fitting model (white dashed line). The gray shaded area gives the uncertainty of the best-fitting model in terms of percentage.

The best-fitting solution gives a total mass of the stellar clusters of $1.6_{\vee 0.9}^{\wedge 1.9} \times 10^{6} M_{\odot}$. Taking into account the number of SF regions detected we can estimate an average value of about $5 \times 10^{3} M_{\odot}$ for the individual stellar clusters, well centered within the limits estimated by Sharma et al. (2011). The dust luminosity associated with the SF regions is $0.42 \wedge 0.49 \times 10^{9} L_{\odot}$. As Fig. 5 shows, the Groves et al. (2008) model is not able to reproduce the LABOCA $870 \mu \mathrm{m}$ observations (see Table 4 for details). A complete discussion of this excess is given in Sect. 6 .

\subsection{Best fit for the diffuse component}

We searched the library of the diffuse dust SEDs of Popescu et al. (2011) for the best-fitting solution to the data in the MIR/submm range, leaving $\tau_{B}^{\mathrm{f}}$ and $S F R^{\prime}$ as free parameters and keeping old fixed to 0.05 and $B / D$ to 0.0 (see Table 3 for details). For each of the 100 iterations we tested a total of 784 different combinations of $\tau_{B}^{\mathrm{f}}$ and $S F R^{\prime}$. The flux level was scaled using Eq. (8) and $h_{\mathrm{s}}=2513 \mathrm{pc}$. As we mentioned in Sect. 3, Planck data were not used in the fitting procedure since their diffuse fluxes were not directly measured but inferred, while the LABOCA $870 \mu \mathrm{m}$ band was not used owing to the observational limitations discussed in Sect. 2.6. The $\mathrm{S} 36 \mathrm{~mm}$ data point was also not used since the thermal radio continuum is not related to the diffuse ISM but to the ionized gas within the SF component. The results are shown in Fig. 6 and Table 4. The reduced $\chi^{2}$ is $1.82_{\vee}^{\wedge} 4.44$ and the dust luminosity associated with the diffuse component is $1.30_{\vee 1.08}^{\wedge 1.50} \times 10^{9} L_{\odot}$.

The model of Popescu et al. (2011) systematically underpredicts the submm and mm estimated fluxes from Planck (see Table 4 for details). This excess of emission is discussed in Sect. 6.

\subsection{Total emission}

Once we separately fitted the emission from the SF and the diffuse component, we combined both SEDs to match the total emission of the galaxy, which is shown in Fig. 7. At MIR/FIR wavelengths the observations are reasonably well fitted, while at submm and $\mathrm{mm}$ wavelengths the excess of emission found in the individual components translates into a significant excess in the global SED of M 33 (Fig. 7), showing up at submm wavelengths and reaching compelling values at $\mathrm{mm}$ wavelengths (see Table 4). It is important to note that no traces of the excess are visible at the Planck $10 \mathrm{~mm}$ point extracted from the Planck Catalogue of Compact Sources (see Sect. 2.7).

From the values that we obtained for the radiation field leaving the SF regions and powering the diffuse dust emission, $S F R^{\prime}$, and for the average fraction of the SF component covered by PDRs, $f_{\text {cov }}$, we estimated the intrinsic SFR of M 33 as

$S F R=\frac{S F R^{\prime}}{1-f_{\text {cov }}}=0.45_{\vee 0.31}^{\wedge 1.13} M_{\odot} \mathrm{yr}^{-1}$,

in good agreement with the value $0.45 \pm 0.10 \mathrm{M}_{\odot} \mathrm{yr}^{-1}$ found by Verley et al. (2009) using extinction corrected UV and $\mathrm{H} \alpha$ measurements.

\subsection{Energetic balance}

The comparison between the predicted radiation field escaping M 33 (see Sect. 4.1 for details) and the observations is shown in Fig. 8. The attenuation and its uncertainty were calculated using $\tau_{B}^{\mathrm{f}}=2.2_{\vee 1.6}^{\wedge} 2.8$ and $f_{\text {cov }}=0.46_{\vee 0.36}^{\wedge 0.74}$ as explained in Sect. 4.1 . There is a good agreement in the infrared part because the parameter old was scaled by hand to match $S_{v}^{\text {s,old }}$ to the observations (see 


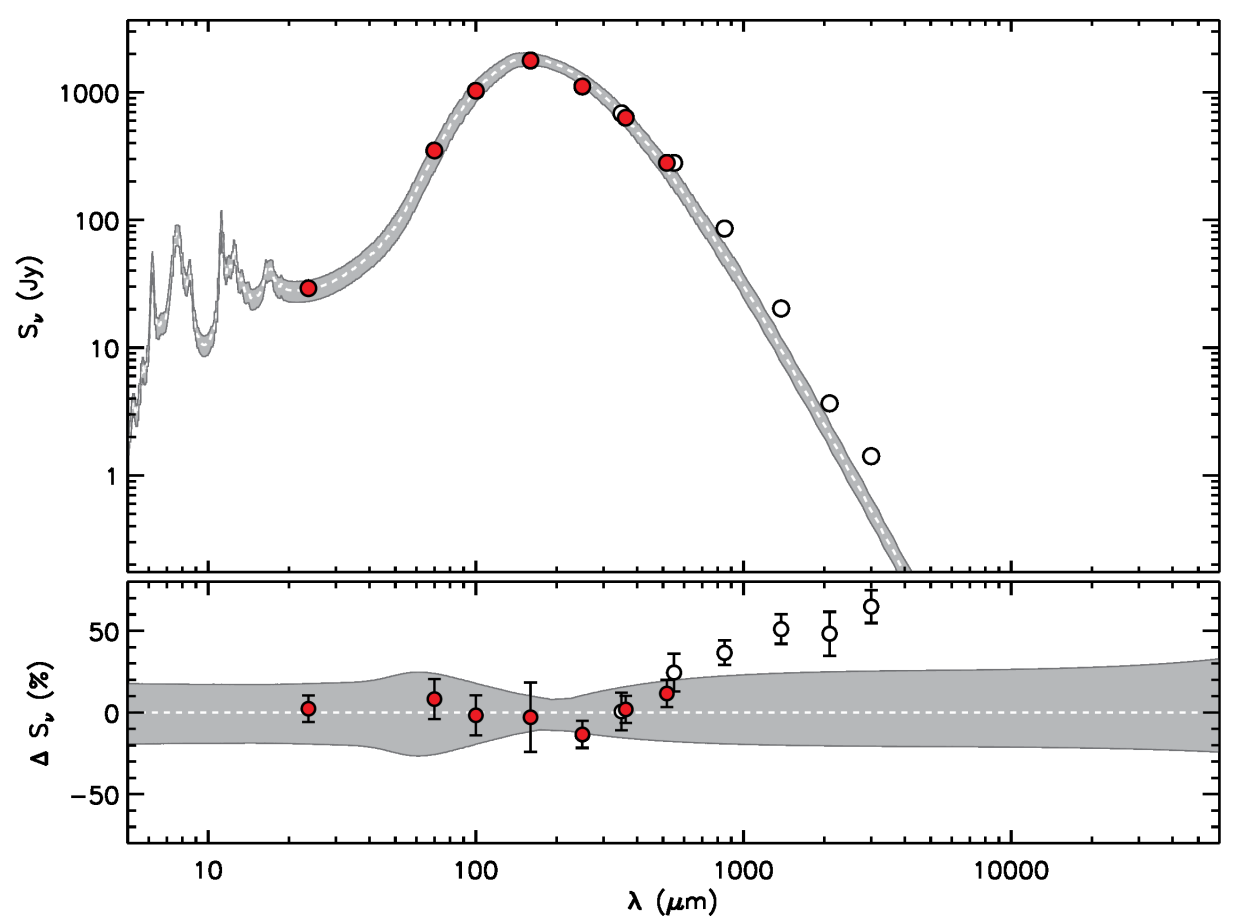

Fig. 6. Top: results of the fitting procedure for the diffuse component of M33. The red filled circles are the observed fluxes for the diffuse component presented in Table 2 . The best-fitting model is represented by the white dashed line while its uncertainty is defined by the gray shaded area. For clarity, errors are only shown in the bottom panel. Bottom: residuals of the fitting procedure. The red filled circles correspond to the percentage difference of the observed fluxes and the best-fitting model (white dashed line). The gray shaded area gives the uncertainty of the best-fitting model in terms of percentage.

Table 4. Comparison of the observations and the models for M 33.

\begin{tabular}{|c|c|c|c|c|c|c|}
\hline BAND & $\frac{S_{\mathrm{SF}}^{\mathrm{obs}}}{S_{\mathrm{SF}}^{\text {mod }}}$ & $\frac{\Delta_{\mathrm{SF}}}{\sqrt{\left(\delta_{\mathrm{SF}}^{\mathrm{obs}}\right)^{2}+\left(\delta_{\mathrm{SF}}^{\mathrm{mod}}\right)^{2}}}$ & $\frac{S_{\mathrm{DDF}}^{\mathrm{obs}}}{S_{\mathrm{DIFF}}^{\operatorname{mmod}}}$ & $\frac{\Delta_{\mathrm{DIFF}}}{\sqrt{\left(\delta_{\mathrm{DIFF}}^{\text {obs }}\right)^{2}+\left(\delta_{\mathrm{DIFF}}^{\mathrm{mod}}\right)^{2}}}$ & $\frac{S_{\mathrm{TOT}}^{\text {obs }}}{S_{\mathrm{TOT}}^{\text {mod }}}$ & $\frac{\Delta_{\mathrm{TOT}}}{\sqrt{\left(\delta_{\mathrm{TOT}}^{\text {obs }}\right)^{2}+\left(\delta_{\mathrm{TOT}}^{\mathrm{mod}}\right)^{2}}}$ \\
\hline GALEX FUV & - & - & - & - & $4.28_{\vee 2.64}^{\wedge 197.88}$ & 5.22 \\
\hline GALEX NUV & - & - & - & - & $3.98_{\vee 2.91}^{\wedge} 8.18$ & 7.76 \\
\hline Sloan $u$-band & - & - & - & - & $1.68 \wedge 2.35$ & 2.70 \\
\hline MIPS $24 \mu \mathrm{m}$ & $1.11_{\vee} 1.35$ & 0.44 & $1.02 \wedge 1.27$ & 0.12 & $1.03 \wedge 1.27$ & 0.17 \\
\hline PACS $70 \mu \mathrm{m}$ & $0.98 \wedge 1.09$ & -0.13 & $1.09 \stackrel{\wedge}{\wedge 1.47}$ & 0.33 & $1.05 \vee 1.30$ & 0.24 \\
\hline PACS $100 \mu \mathrm{m}$ & $0.99 \wedge 1.13$ & -0.03 & $0.98 \wedge 1.22$ & -0.07 & $0.99 \wedge 1.20$ & -0.07 \\
\hline PACS $160 \mu \mathrm{m}$ & $1.01 \wedge 1.16$ & 0.04 & $0.97 \vee 1.10$ & -0.12 & $0.98_{\vee 0.88}^{\wedge}$ & -0.08 \\
\hline SPIRE $250 \mu \mathrm{m}$ & $0.99 \wedge 1.17$ & -0.04 & $0.88_{\vee}^{\wedge} 1.01$ & -0.78 & $0.90 \wedge 1.04$ & -0.68 \\
\hline Planck $350 \mu \mathrm{m}$ & - & - & $1.01 \wedge 1.19$ & 0.03 & $1.01 \wedge 1.19$ & 0.03 \\
\hline SPIRE $350 \mu \mathrm{m}$ & $1.08_{\vee 0.91}^{\wedge 1.32}$ & 0.42 & $1.02 \wedge 1.21$ & 0.11 & $1.02 \wedge 1.21$ & 0.12 \\
\hline SPIRE $500 \mu \mathrm{m}$ & $1.25 \vee 1.57$ & 1.26 & $1.13 \wedge 1.37$ & 0.63 & $1.15_{\vee 0.97}^{\wedge}$ & 0.74 \\
\hline Planck $550 \mu \mathrm{m}$ & - & - & $1.32 \wedge 1.61$ & 1.41 & $1.29 \wedge 1.57$ & 1.27 \\
\hline Planck $850 \mu \mathrm{m}$ & - & - & $1.58_{\vee 1.29}^{\wedge}$ & 2.45 & $1.52 \wedge 1.88$ & 2.23 \\
\hline LABOCA $870 \mu \mathrm{m}$ & $1.75_{\vee 1.47}^{\wedge}$ & 3.43 & - & - & - & - \\
\hline Planck $1.4 \mathrm{~mm}$ & - & - & $2.04 \wedge 2.55$ & 4.07 & $1.91 \wedge 1.39$ & 3.66 \\
\hline Planck $2.1 \mathrm{~mm}$ & - & - & $1.93 \wedge 1.55$ & 3.29 & $1.73_{\vee}^{\wedge} 2.151$ & 2.74 \\
\hline Planck $3.0 \mathrm{~mm}$ & - & - & $2.84 \vee 3.58$ & 6.78 & $1.98_{\vee}^{\wedge} 2.44$ & 4.24 \\
\hline Planck $10 \mathrm{~mm}$ & - & - & - & - & $0.86_{\vee 0.74}^{\wedge}$ & -0.48 \\
\hline $\mathrm{S} 36 \mathrm{~mm}$ & $0.81 \wedge 0.99$ & -0.86 & - & - & $0.81_{\vee 0.70}^{\wedge 0.99}$ & -0.86 \\
\hline
\end{tabular}

Notes. The band names are specified in Col. 1. Columns 2 and 3 show the results obtained for the SF component, Cols. 4 and 5 for the diffuse component, and Cols. 6 and 7 for the total emission. In the three cases $\Delta$ gives the deviation of the observed data points from the model expectations, while $\delta^{\text {obs }}$ and $\delta^{\text {mod }}$ corresponds to the relative error of the observations and the models, respectively. 


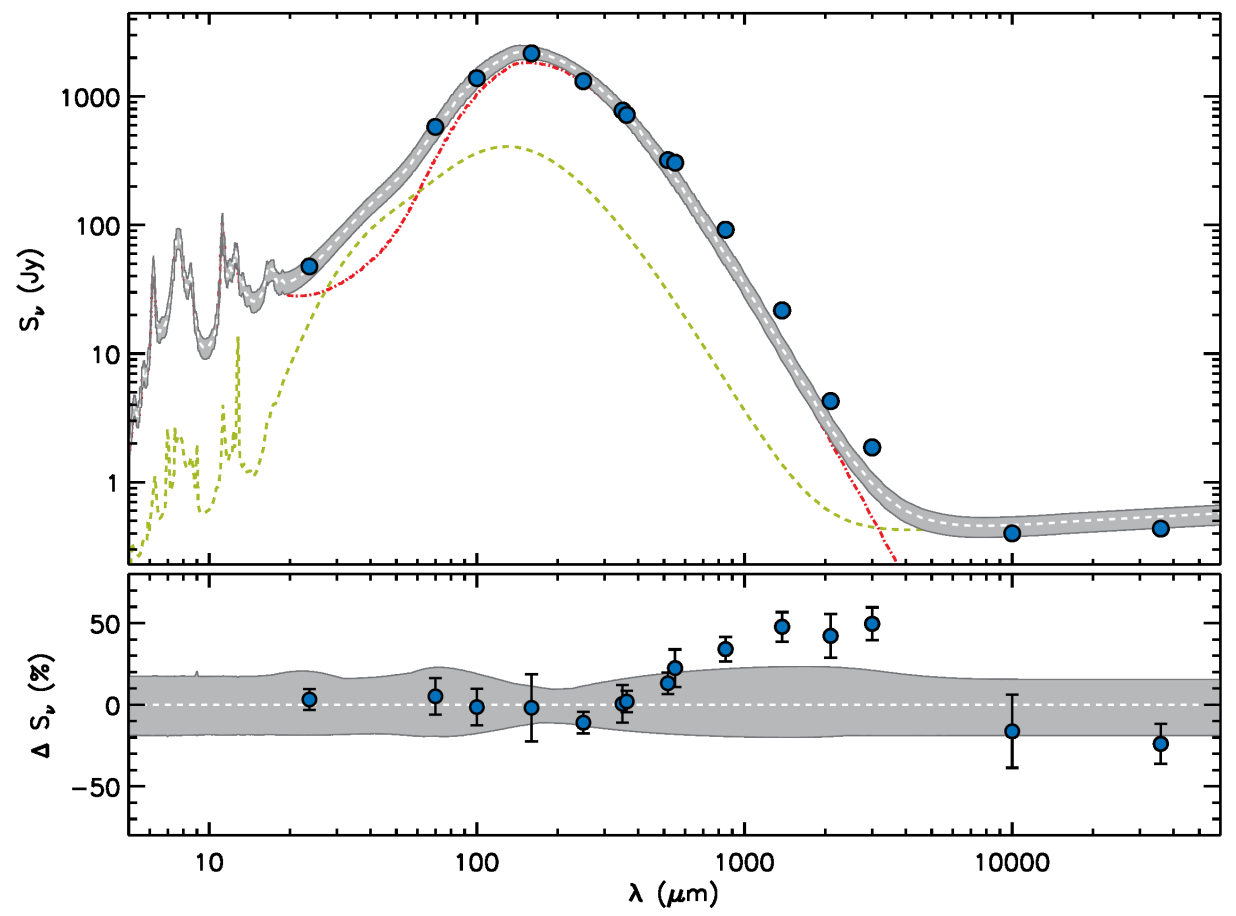

Fig. 7. Top: combination of the best-fitting models for the SF component (green dashed line, see Fig. 5 for details) and the diffuse component (red dashed-dotted line, see Fig. 6 for details). The blue filled circles are the observed fluxes for the entire galaxy presented in Table 2. The white dashed line and the gray shaded area correspond to the direct sum of the best-fitting models and their uncertainties obtained for the SF and the diffuse components. For clarity, errors are only shown in the bottom panel. Bottom: residuals of the fitting procedure. The blue filled circles correspond to the percentage difference of the observed fluxes and the best-fitting model (white dashed line). The gray shaded area gives the uncertainty of the best-fitting model in terms of percentage.

Sect. 4.3). On the other hand, in the UV-optical part we found that the integrated luminosity calculated from the observations is $70 \wedge 84 \%$ higher than the expected luminosity. This discrepancy is discussed in Sect. 6.

\subsection{Gas-to-dust ratio}

In the Groves et al. (2008) model, the mass of dust contained in the SF regions depends on the combination of parameters and it scales with $M_{\mathrm{cl}}$. For the SF component of M 33 we obtained

$M_{\text {dust }}^{\mathrm{SF}}=2.9_{\vee 2.5}^{\wedge 3.5} \times 10^{6} M_{\odot}$.

The total mass of diffuse dust of M 33 can be calculated from the Eq. (44) of Popescu et al. (2011):

$M_{\text {dust }}^{\text {diff }}=0.992 \times \tau_{B}^{\mathrm{f}} \times h_{\mathrm{s}}{ }^{2} \mathrm{pc}^{-2} M_{\odot}=13.8_{\vee 10.0}^{\wedge 17.5} \times 10^{6} M_{\odot}$.

Druard et al. (2014) estimated that the total molecular mass of $\mathrm{M} 33$ is $0.3 \times 10^{9} M_{\odot}$ using a CO-to- $\mathrm{H}_{2}$ conversion factor $X_{\mathrm{CO}}=N\left(\mathrm{H}_{2}\right) / I_{\mathrm{CO}(1 \rightarrow 0)}=4 \times 10^{20} \mathrm{~cm}^{-2} /\left(\mathrm{K} \mathrm{km} \mathrm{s}^{-1}\right)$, including helium. Gratier et al. (2010) used Very Large Array (VLA) observations of the atomic gas and derived a total mass of $1.4 \times 10^{9} M_{\odot}$ within a $8.5 \mathrm{kpc}$ radius. From all these values we can derive a gas-to-dust ratio of

$G_{\mathrm{dust}}=\frac{M_{\mathrm{atom}}+M_{\mathrm{mol}}}{M_{\mathrm{dust}}^{\mathrm{SF}}+M_{\mathrm{dust}}^{\mathrm{diff}}}=\frac{1.7 \times 10^{9} M_{\odot}}{16.7_{\vee 12.5}^{\wedge 21.0} \times 10^{6} M_{\odot}}=101_{\vee 80}^{\wedge 135}$

at the low end of the range determined by Kramer et al. (2010), who found, using MBB models, that $G_{\text {dust }}$ ranges from 200 to 120 depending on the value of $\beta$.
Given the solar value $G_{\text {dust }}=137$ (Draine et al. 2007, Table 2), and assuming that the gas-to-dust mass ratio scales linearly with metallicity (i.e., the fraction of metals incorporated in the dust is constant), owing to the subsolar metallicity of M 33 we would expect a higher value of $G_{\text {dust }}$ between 200 and 450 (see Sect. 4.3 for details). Possible origins for this discrepancy are discussed in Sect. 6.

\section{Discussion}

Our study has revealed three compelling discrepancies between the models and the observations: an excess of emission at submm and mm wavelengths (Fig. 7), a deficit of absorption of UV photons (Fig. 8), and an abnormally low value of $G_{\text {dust }}$ (Sect. 5.5). In the following, we discuss in detail different hypotheses that could explain these discrepancies.

\subsection{Cold dust component}

A population of very cold dust (VCD) with temperatures below $10 \mathrm{~K}$ has been proposed to explain the excess of emission at submm wavelengths (e.g., Galliano et al. 2003, 2005; Galametz et al. 2009, 2010). Although in general the VCD component is able to fit the submm excess well, this hypothesis is considered unlikely because of the large mass of dust required and the strong shielding from the ambient radiation field necessary to keep the grains at such low temperatures (Lisenfeld et al. 2002). In this line, Galliano et al. (2011) found that the submm excess in the LMC can not be produced by a cold dust component. 


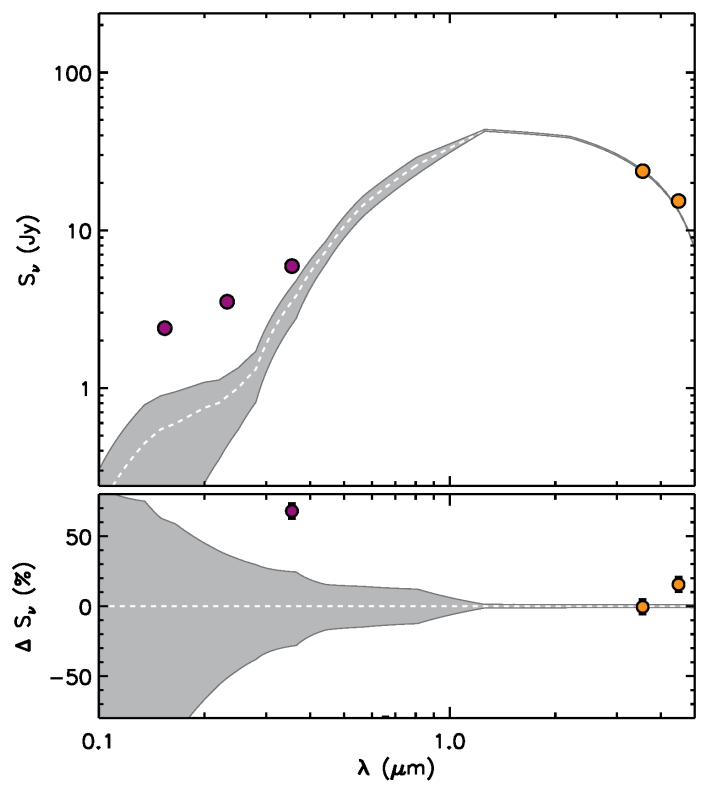

Fig. 8. Top: results of the fitting procedure for the stellar component of M33. The purple and orange filled circles are respectively the observed fluxes of the young and the old stellar populations presented in Table 2. The white dashed line and the gray shaded area correspond to the combination of the attenuated emission and uncertainty of the young and old stellar populations ( $S_{v}^{\text {s,young }}$ and $S_{v}^{\text {s,old }}$, respectively). For clarity, errors are only shown in the bottom panel. Bottom: residuals of the fitting procedure. The filled circles correspond to the percentage difference of the observed fluxes and the best-fitting model (white dashed line). The gray shaded area gives the uncertainty of the best-fitting model in terms of percentage.

To explore the VCD hypothesis in the case of M 33 we added a MBB component with $\beta=2$ to its total SED (see top panel of Fig. 9). We found that the submm and mm excess can be well fitted using a temperature of $\sim 6 \mathrm{~K}$. The mass of dust required to produce such emission is

$M_{\mathrm{VCD}} \sim 40 \times 10^{6} M_{\odot}$,

which is about three times higher than the combined mass of dust found in the SF and the diffuse components. Adding this extra mass of dust would imply an unreasonably low value of $G_{\text {dust }} \lesssim 50$. For reference, we note that Baes et al. (2014) found a value of $G_{\text {dust }}<14.5$ for the extreme case of NGC 5485, an early-type galaxy with no atomic or molecular gas detected but with a prominent dust lane perpendicular to the photometric major axis.

\subsection{Spinning grains}

Draine \& Lazarian (1998) proposed that electric dipole radiation from spinning grains are responsible for the anomalous emission of the diffuse Galactic background peaking near $10 \mathrm{~mm}$. In the Draine \& Lazarian (1998) model the very small grains are disk shaped while large grains are spherical, and both rotate around their axis of major inertia, which means that the frequency of photons emitted is identical to the angular frequency. According to these authors, the rotational excitation of the spinning grains is dominated by collisions with ions and plasma drag and the rotation rate depends on several factors such as the intensity of the radiation field and the physical parameters of the gas
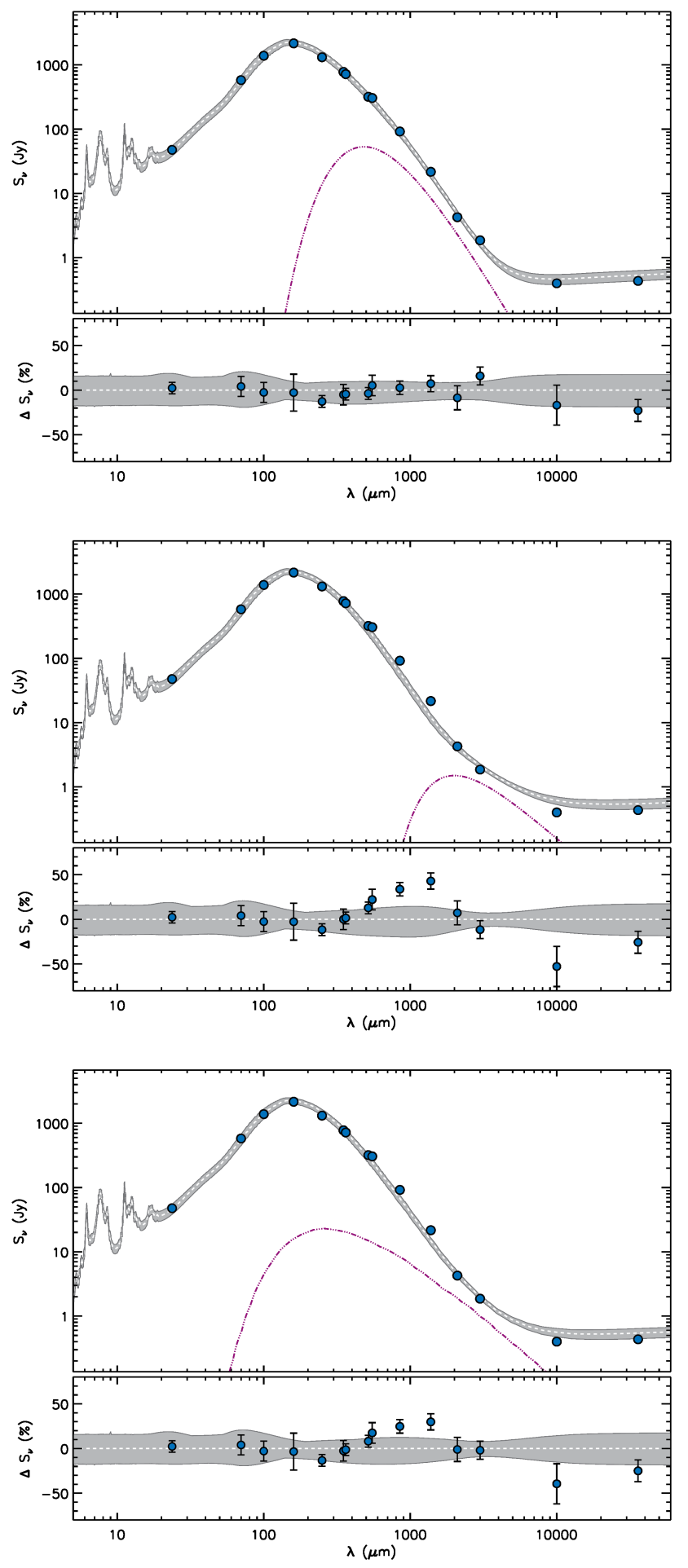

Fig. 9. Total SED of M 33 (see Fig. 7 for a detailed description) combined with an extra component (purple dashed line) to account for the emission from very cold dust (top panel), spinning dust grains (mid panel), and magnetic nanoparticles (bottom panel).

phase. For example, while the peak of the emission from spinning grains ranges from $\sim 7$ to $15 \mathrm{~mm}$ in the cold neutral, warm neutral, and warm ionized medium, it can reach values below $2 \mathrm{~mm}$ in PDRs (see Hoang et al. 2010 for a refined version of the model of Draine \& Lazarian 1998).

To study the possibility that spinning dust grains are responsible for the excess of emission detected in M33, we included 
their contribution in the total SED. As the mid panel of Fig. 9 shows, the excess of emission at wavelengths $\lesssim 2 \mathrm{~mm}$ can not be reproduced even if we assume spinning grains rotating at the highest rate allowed by Hoang et al. (2010). Moreover, the contribution from spinning grains degrades the good agreement previously found between models and observations at wavelengths around $10 \mathrm{~mm}$.

\subsection{Magnetic nanoparticles}

Draine \& Hensley (2013) claimed that if part of the interstellar Fe forms ferro- or ferri-magnetic grains, then the magnetic dipole radiation from these grains might contribute significantly to the total dust SED. These authors considered three materials as potential candidates: metallic $\mathrm{Fe}$, magnetite $\left(\mathrm{Fe}_{3} \mathrm{O}_{4}\right)$, and maghemite $\left(\gamma-\mathrm{Fe}_{2} \mathrm{O}_{3}\right)$. Draine \& Hensley (2012) show how magnetic grains are able to explain the strong excess of submm and $\mathrm{mm}$ emission found in the SMC.

In order to test whether magnetic nanoparticles can explain the excess of emission found in M 33, we fitted the SED expected for $10 \mathrm{~nm}$ metallic $\mathrm{Fe}$ grains at $18 \mathrm{~K}$ (see right panel in Fig. 8 of Draine \& Hensley 2013) by adjusting the intensity until the best fit to the FIR-mm data points was achieved, as is shown in the bottom panel of Fig. 9. The contribution from magnetic grains can alleviate the $\mathrm{mm}$ and submm excess but is not able to satisfactorily fit the data in this wavelength range.

We also tested the possibility of $100 \mathrm{~nm}$ metallic Fe grains at $18 \mathrm{~K}$. The fit is worse than for the $10 \mathrm{~nm}$ grains in the subm$\mathrm{m} / \mathrm{mm} /$ Planck $10 \mathrm{~mm}$ range. In addition, they have a strong impact on the peak of the SED thus degrading the fit at wavelengths $\sim 150 \mu \mathrm{m}$. Thus, for M 33, in contrast to the SMC, magnetic grain emission is not an entirely satisfactory explanation for the $\mathrm{mm}$ and submm excess.

\subsection{CO-to- $\mathrm{H}_{2}$ conversion factor}

The low value of $G_{\text {dust }}$ obtained here depends in part on the mass of molecular gas obtained by Druard et al. (2014), who determined a conversion factor $X_{\mathrm{CO}}=4 \times 10^{20} \mathrm{~cm}^{-2} /\left(\mathrm{K} \mathrm{km} \mathrm{s}^{-1}\right)$ for M33, about two times higher than the Galactic value, which is reasonable for the metallicity that is approximately two times lower. A value of $X_{\mathrm{CO}} \gtrsim 20 \times 10^{20} \mathrm{~cm}^{-2} /\left(\mathrm{K} \mathrm{km} \mathrm{s}^{-1}\right)$ would provide enough molecular gas to achieve a $G_{\text {dust }}$ in the expected range of $G_{\text {dust }} \gtrsim 200$. However, this is an unreasonably high value for the case of M33, where after combining $\mathrm{CO}, \mathrm{HI}$, and IR measurements, Leroy et al. (2011) did not find evidence of such deviations in the value of $X_{\mathrm{CO}}$.

\subsection{Porosity}

A porous ISM would allow a higher fraction of the UV radiation to escape the galaxy without interacting with the dust thus alleviating the UV discrepancy. Several theoretical studies try to explain how the internal properties of galaxies affect the escape fraction. Among the properties that can influence the escape fraction are the covering factor of the clumps in the ISM, as well as the density of the clumped and inter clump medium (Fernandez \& Shull 2011), blow-out of shells created by supernova $(\mathrm{SN})$ remnants that provide the photons with a clear path to escape the galaxy (Dove \& Shull 1994), and the porosity of the ISM given as the fraction of the ISM that is devoid of H I owing to the expansion of SN-driven bubbles. Therefore, the UV discrepancy derived from our modeling might be due to large shell structures across the whole galactic disk (Relaño et al. 2013).

\subsection{Dust heated by evolved stars}

Could an unrealistic SED of the stellar populations that are heating the dust be responsible for the $\mathrm{mm}$ and submm excess and/or the UV discrepancy? Popescu et al. (2011) discussed in detail the influence of the spectral shape of the stellar radiation on the dust SED (see their Sect. 4.3). They concluded that the stellar SED does not significantly affect the shape of the dust SED and that the most relevant parameters shaping the dust SED are the overall stellar luminosities as well as the geometry and the properties of the dust. Therefore, the exact shape of the stellar SED is unlikely to be a very important source of error in our study.

In order to quantify in M33 the contribution to the dust heating by the evolved stellar population, we set $S F R$ to 0 and checked the amplitude of the diffuse SED keeping the remaining values the same as in our best-fitting solution. We found that this contribution is indeed negligible with the exception of the wavelengths around the peak, where it contributes slightly to the dust emission. It is noteworthy that this result is at first sight in contradiction with the results of Boquien et al. (2011), who found a tight correlation between the $250 / 350 \mu \mathrm{m}$ ratio and the $3.6 \mu \mathrm{m}$ luminosity $\left(L_{3.6 \mu \mathrm{m}}\right)$ at scales of $42^{\prime \prime}(\sim 170 \mathrm{pc})$, which they interpret as an indication that the old stars are the main heating source of the dust emitting in the $250-350 \mu \mathrm{m}$ wavelength range. This apparent discrepancy between our study and the Boquien et al. (2011) study is not real, but it comes from different definitions of what is considered an "old" stellar population (see our Sect. 4.1 for details).

\subsection{Geometry}

The discrepancies revealed by our study might be explained by differences between the actual geometry of M 33 and the geometry assumed by the model of Popescu et al. (2011, Table E.1). As we mentioned in Sect. 4.3, there is no evidence of any discrepancy regarding the radial dimensions of the disk of dust. Regarding the vertical dimensions, for a $B$-band scalelength of $2513 \mathrm{pc}$, the model of Popescu et al. (2011) predicts scaleheights of $186 \mathrm{pc}$ for the old stellar disk, $121 \mathrm{pc}$ for the thick disk of dust, and $40 \mathrm{pc}$ for both the young stellar disk and the thin disk of dust. Using the break of the power-spectrum in different emission bands, Combes et al. (2012) were able to give an estimation of the thickness of the stellar and dust disks of M 33: cold dust lies in a $350 \mathrm{pc}$ disk, warm dust lies in a $100 \mathrm{pc}$ disk, and the thickness of the stellar disk in the FUV and NUV bands was found to be $40 \mathrm{pc}$ and $56 \mathrm{pc}$, respectively. Unfortunately, the conversion of the breaks found by Combes et al. (2012) to scaleheights is not straightforward since it depends on the conditions in the disk as the simulations of Combes et al. (2012, Table 4) have shown. Therefore, we can not exclude that the discrepancies found in our study are due to differences between the vertical dimensions of M 33 and the model assumptions.

\subsection{Different dust properties}

Differences between the actual dust properties of the low metallicity galaxy M33 and those adopted in the models (Laor \& Draine 1993; Li \& Draine 2001; Weingartner \& Draine 2001; Draine \& Li 2007) might simultaneously explain the three discrepancies found. Recently Jones et al. (2013) proposed that 
interstellar dust in the diffuse ISM might be characterized by a lower emissivity index $\beta$ at long wavelengths. If this is the case, these dust grains would provide flatter emission at submm and $\mathrm{mm}$ wavelengths, which would result in a better agreement between our modeling and the observations. Additionally, a lower emissivity index resulting in a higher extinction coefficient would allow a lower dust surface density to account for the dust emission, which would result in a decrement of the UV absorption, and thus a lower UV excess, and a lower dust mass, thus increasing the value of $G_{\text {dust }}$.

\section{Summary and conclusions}

We have modeled separately the emission coming from the SF regions and from the diffuse ISM in the spiral galaxy M33, using radiation transfer models based on dust grains with standard properties. Thanks to the wide set of data available for this galaxy, as well as the numerous previous studies from other authors, we were able to constrain an important part of the parameters used by the models.

For the SF component, we found that the Groves et al. (2008) model is able to reproduce the MIR-FIR observations and the thermal radio continuum. However, we found that the model underestimates the LABOCA data and it is barely compatible with the SPIRE $500 \mu \mathrm{m}$ data. Regarding the diffuse component, we found that the Popescu et al. (2011) model fits the MIR/FIR data points well but severely underpredicts the observations longwards $800 \mu \mathrm{m}$. We also found that our modeling underpredicts by $70 \wedge 84 \%$ the UV radiation that escapes the galaxy without interacting with the dust. We derived a gas-to-dust ratio $G_{\text {dust }}=101 \wedge 135$, significantly lower than the expected value based on the subsolar metallicity of M 33 .

We discussed various processes that can explain these discrepancies. A cold dust component emitting in the submm and $\mathrm{mm}$ is unlikely owing to the large mass of dust that would be needed. Spinning grains and the magnetic nanoparticles can not reproduce well the submm and $\mathrm{mm}$ excess and they seriously degrade the fit at other wavelengths. Previous studies of the molecular gas in M 33 discards the possibility of anomalies in the $X_{\mathrm{CO}}$ conversion factor that can explain the low value of $G_{\mathrm{dust}}$. Since in the model of Popescu et al. (2011) the exact shape of the stellar radiation field has little influence on the dust emission, dust heating from evolved stars is unlikely to be a source of error. Differences in the geometry assumed by the model and the actual geometry of M33 can explain the discrepancies, but there is no observational evidence for such differences. A porous ISM can explain the excess escape of UV radiation.

As a final option, we discussed different physical properties of the dust grains. A lower $\beta$ would flatten the dust emission at submm and mm wavelengths, decrease the UV attenuation, and bring the gas-to-dust ratio closer to the expected value, thus explaining simultaneously the three discrepancies.

Acknowledgements. We thank the second referee for the constructive report and helpful suggestions. We thank F.-X. Désert for his invaluable help with the Planck data. This work was partially supported by a Junta de Andalucía Grant FQM108, a Spanish MEC Grant AYA-2011-24728 and AYA2014-53506-P, Juan de la Cierva fellowship Program and the European Reintegration Grant ERG HER-SFR. T.R.L. is grateful for the support of the Spanish Ministerio de Educación, Cultura y Deporte by means of the FPU fellowship. This publication was financed by the FIC-R Fund, allocated to the project 30321072. M.A. acknowledges support by the German Research Foundation (DFG) in the framework of the priority program 1573, "Physics of the Interstellar Medium", through grant number AL 1467/2-1. This research made use of the NASA/IPAC Extragalactic Database (NED), which is operated by the Jet Propulsion Laboratory, California Institute of Technology, under contract with the National Aeronautics and Space Administration. We also acknowledge the use of the HyperLeda database (http://leda.univ-lyon1.fr). Funding for the creation and distribution of the SDSS Archive has been provided by the Alfred P. Sloan Foundation, the Participating Institutions, the National Aeronautics and Space Administration, the National Science Foundation, the US Department of Energy, the Japanese Monbukagakusho, and the Max Planck Society. The SDSS Web site is http://www.sdss.org/. The SDSS is managed by the Astrophysical Research Consortium (ARC) for the Participating Institutions. The Participating Institutions are The University of Chicago, Fermilab, the Institute for Advanced Study, the Japan Participation Group, The Johns Hopkins University, the Korean Scientist Group, Los Alamos National Laboratory, the Max-Planck-Institute for Astronomy (MPIA), the Max-Planck-Institute for Astrophysics (MPA), New Mexico State University, University of Pittsburgh, University of Portsmouth, Princeton University, the United States Naval Observatory, and the University of Washington. This research made use of Montage, funded by the National Aeronautics and Space Administration's Earth Science Technology Office, Computational Technnologies Project, under Cooperative Agreement Number NCC5-626 between NASA and the California Institute of Technology. The code is maintained by the NASA/IPAC Infrared Science Archive. This research made use of python (http: //www . python. org), of Matplotlib (Hunter 2007), a suite of open-source python modules that provides a framework for creating scientific plots, and Astropy, a community-developed core Python package for Astronomy (Astropy Collaboration et al. 2013). This research made use of APLpy, an opensource plotting package for Python hosted at http://aplpy.github.com.

\section{References}

Abazajian, K. N., Adelman-McCarthy, J. K., Agüeros, M. A., et al. 2009, ApJS, 182,543

Aniano, G., Draine, B. T., Gordon, K. D., \& Sandstrom, K. 2011, PASP, 123, 1218

Asplund, M., Grevesse, N., \& Sauval, A. J. 2005, in Cosmic Abundances as Records of Stellar Evolution and Nucleosynthesis, eds. T. G. Barnes, III, \& F. N. Bash, ASP Conf. Ser., 336, 25

Baes, M., Allaert, F., Sarzi, M., et al. 2014, MNRAS, 444, L90

Bendo, G. J., Buckalew, B. A., Dale, D. A., et al. 2006, ApJ, 645, 134

Bendo, G. J., Griffin, M. J., Bock, J. J., et al. 2013, MNRAS, 433, 3062

Bendo, G. J., Baes, M., Bianchi, S., et al. 2015, MNRAS, 448, 135

Bertin, E., \& Arnouts, S. 1996, A\&AS, 117, 393

Boquien, M., Calzetti, D., Kramer, C., et al. 2010, A\&A, 518, L70

Boquien, M., Calzetti, D., Combes, F., et al. 2011, AJ, 142, 111

Boquien, M., Calzetti, D., Aalto, S., et al. 2015, A\&A, 578, A8

Bot, C., Ysard, N., Paradis, D., et al. 2010, A\&A, 523, A20

Braine, J., Gratier, P., Kramer, C., et al. 2010, A\&A, 520, A107

Combes, F., Boquien, M., Kramer, C., et al. 2012, A\&A, 539, A67

Corbelli, E., \& Walterbos, R. A. M. 2007, ApJ, 669, 315

Dale, D. A., Aniano, G., Engelbracht, C. W., et al. 2012, ApJ, 745, 95

Deul, E. R., \& van der Hulst, J. M. 1987, A\&AS, 67, 509

Dove, J. B., \& Shull, J. M. 1994, ApJ, 430, 222

Draine, B. T., \& Hensley, B. 2012, ApJ, 757, 103

Draine, B. T., \& Hensley, B. 2013, ApJ, 765, 159

Draine, B. T., \& Lazarian, A. 1998, ApJ, 508, 157

Draine, B. T., \& Li, A. 2007, ApJ, 657, 810

Draine, B. T., Dale, D. A., Bendo, G., et al. 2007, ApJ, 663, 866

Druard, C., Braine, J., Schuster, K. F., et al. 2014, A\&A, 567, A118

Engelbracht, C. W., Blaylock, M., Su, K. Y. L., et al. 2007, PASP, 119, 994

Ercolano, B., Barlow, M. J., \& Storey, P. J. 2005, MNRAS, 362, 1038

Fan, Z., \& de Grijs, R. 2014, ApJS, 211, 22

Fazio, G. G., Hora, J. L., Allen, L. E., et al. 2004, ApJS, 154, 10

Fernandez, E. R., \& Shull, J. M. 2011, ApJ, 731, 20

Freedman, W. L., Wilson, C. D., \& Madore, B. F. 1991, ApJ, 372, 455

Galametz, M., Madden, S., Galliano, F., et al. 2009, A\&A, 508, 645

Galametz, M., Madden, S. C., Galliano, F., et al. 2010, A\&A, 518, L55

Galametz, M., Madden, S. C., Galliano, F., et al. 2011, A\&A, 532, A56

Galametz, M., Albrecht, M., Kennicutt, R., et al. 2014, MNRAS, 439, 2542

Galliano, F., Madden, S. C., Jones, A. P., et al. 2003, A\&A, 407, 159

Galliano, F., Madden, S. C., Jones, A. P., Wilson, C. D., \& Bernard, J.-P. 2005 A\&A, 434, 867

Galliano, F., Hony, S., Bernard, J.-P., et al. 2011, A\&A, 536, A88

Gil de Paz, A., Boissier, S., Madore, B. F., et al. 2007, ApJS, 173, 185

Gordon, K. D., Roman-Duval, J., Bot, C., et al. 2014, ApJ, 797, 85

Górski, K. M., Hivon, E., Banday, A. J., et al. 2005, ApJ, 622, 759

Gratier, P., Braine, J., Rodriguez-Fernandez, N. J., et al. 2010, A\&A, 522, A3

Greenawalt, B. E. 1998, Ph.D. Thesis, New Mexico State University

Griffin, M. J., Abergel, A., Abreu, A., et al. 2010, A\&A, 518, L3

Grossi, M., Corbelli, E., Giovanardi, C., \& Magrini, L. 2010, A\&A, 521, A41

Groves, B., Dopita, M. A., Sutherland, R. S., et al. 2008, ApJS, 176, 438 
Güsten, R., Nyman, L. Å., Schilke, P., et al. 2006, A\&A, 454, L13 Hermelo, I., Lisenfeld, U., Relaño, M., et al. 2013, A\&A, 549, A70 Hippelein, H., Haas, M., Tuffs, R. J., et al. 2003, A\&A, 407, 137 Hoang, T., Draine, B. T., \& Lazarian, A. 2010, ApJ, 715, 1462

Hoopes, C. G., \& Walterbos, R. A. M. 2000, ApJ, 541, 597

Hunter, J. D. 2007, Comput. Sci. Eng., 9, 90

Israel, F. P., Wall, W. F., Raban, D., et al. 2010, A\&A, 519, A67

Jones, A. P., Fanciullo, L., Köhler, M., et al. 2013, A\&A, 558, A62

Kirkpatrick, A., Calzetti, D., Galametz, M., et al. 2013, ApJ, 778, 51

Kramer, C., Buchbender, C., Xilouris, E. M., et al. 2010, A\&A, 518, L67

Laor, A., \& Draine, B. T. 1993, ApJ, 402, 441

Leroy, A. K., Bolatto, A., Gordon, K., et al. 2011, ApJ, 737, 12

Li, A., \& Draine, B. T. 2001, ApJ, 554, 778

Lisenfeld, U., Israel, F. P., Stil, J. M., \& Sievers, A. 2002, A\&A, 382, 860

Magrini, L., Vílchez, J. M., Mampaso, A., Corradi, R. L. M., \& Leisy, P. 2007, A\&A, 470, 865

Makovoz, D., \& Marleau, F. R. 2005, PASP, 117, 1113

Martin, D. C., Fanson, J., Schiminovich, D., et al. 2005, ApJ, 619, L1

Minniti, D., Olszewski, E. W., \& Rieke, M. 1993, ApJ, 410, L79

Morrissey, P., Conrow, T., Barlow, T. A., et al. 2007, ApJS, 173, 682

Ott, S. 2010, in Astronomical Data Analysis Software and Systems XIX, eds. Y. Mizumoto, K.-I. Morita, \& M. Ohishi, ASP Conf. Ser., 434, 139

Ott, S. 2011, in Astronomical Data Analysis Software and Systems XX, eds. I. N Evans, A. Accomazzi, D. J. Mink, \& A. H. Rots, ASP Conf. Ser., 442, 347

Padmanabhan, N., Schlegel, D. J., Finkbeiner, D. P., et al. 2008, ApJ, 674, 1217

Paradis, D., Paladini, R., Noriega-Crespo, A., et al. 2011, ApJ, 735, 6

Planck Collaboration XVII. 2011, A\&A, 536, A17

Planck Collaboration I. 2014, A\&A, 571, A1

Planck Collaboration XIII. 2014, A\&A, 571, A13

Planck Collaboration XXVIII. 2014, A\&A, 571, A28

Planck Collaboration IX. 2014, A\&A, 571, A9
Planck Collaboration XII. 2014, A\&A, 571, A12

Planck Collaboration XXV. 2015, A\&A, 582, A28

Poglitsch, A., Waelkens, C., Geis, N., et al. 2010, A\&A, 518, L2

Popescu, C. C., Tuffs, R. J., Dopita, M. A., et al. 2011, A\&A, 527, A109

Reach, W. T., Megeath, S. T., Cohen, M., et al. 2005, PASP, 117, 978

Regan, M. W., \& Vogel, S. N. 1994, ApJ, 434, 536

Relaño, M., Verley, S., Pérez, I., et al. 2013, A\&A, 552, A140

Rieke, G. H., Young, E. T., Engelbracht, C. W., et al. 2004, ApJS, 154, 25

Robitaille, T. P., Tollerud, E. J., et al. Astropy Collaboration, 2013, A\&A, 558, A33

Roussel, H. 2013, PASP, 125, 1126

Rowan-Robinson, M., Babbedge, T., Oliver, S., et al. 2008, MNRAS, 386, 697

Schuller, F., Menten, K. M., Contreras, Y., et al. 2009, A\&A, 504, 415

Schuster, K.-F., Boucher, C., Brunswig, W., et al. 2004, A\&A, 423, 1171

Serra Díaz-Cano, L., \& Jones, A. P. 2008, A\&A, 492, 127

Sharma, S., Corbelli, E., Giovanardi, C., Hunt, L. K., \& Palla, F. 2011, A\&A, 534, A96

Siringo, G., Kreysa, E., Kovács, A., et al. 2009, A\&A, 497, 945

Tabatabaei, F. S., Beck, R., Krügel, E., et al. 2007a, A\&A, 475, 133

Tabatabaei, F. S., Krause, M., \& Beck, R. 2007b, A\&A, 472, 785

Tabatabaei, F. S., Braine, J., Xilouris, E. M., et al. 2014, A\&A, 561, A95

Thilker, D. A., Hoopes, C. G., Bianchi, L., et al. 2005, ApJ, 619, L67

Tuffs, R. J., Popescu, C. C., Völk, H. J., Kylafis, N. D., \& Dopita, M. A. 2004, A\&A, 419, 821

Verley, S., Hunt, L. K., Corbelli, E., \& Giovanardi, C. 2007, A\&A, 476, 1161 Verley, S., Corbelli, E., Giovanardi, C., \& Hunt, L. K. 2009, A\&A, 493, 453

Verley, S., Corbelli, E., Giovanardi, C., \& Hunt, L. K. 2010a, A\&A, 510, A64

Verley, S., Relaño, M., Kramer, C., et al. 2010b, A\&A, 518, L68

Weingartner, J. C., \& Draine, B. T. 2001, ApJ, 548, 296

Werner, M. W., Roellig, T. L., Low, F. J., et al. 2004, ApJS, 154, 1

Xilouris, E. M., Tabatabaei, F. S., Boquien, M., et al. 2012, A\&A, 543, A74

York, D. G., Adelman, J., Anderson, Jr., J. E., et al. 2000, AJ, 120, 1579 


\section{Appendix A: MBB fit and color corrections}

As pointed out in Sect. 1, MBB models are one of the simplest and most widely used techniques to fit the dust SED. Kramer et al. (2010) found that a MBB with $\beta=1.5$ can reproduce the dust SED of M 33 up to of $500 \mu \mathrm{m}$, the longest wavelength available in their study. Here we confirm that the value $\beta=1.5$ found by these authors is still valid up to wavelengths of $3 \mathrm{~mm}$ as revealed by Planck data (see Fig. A.1).

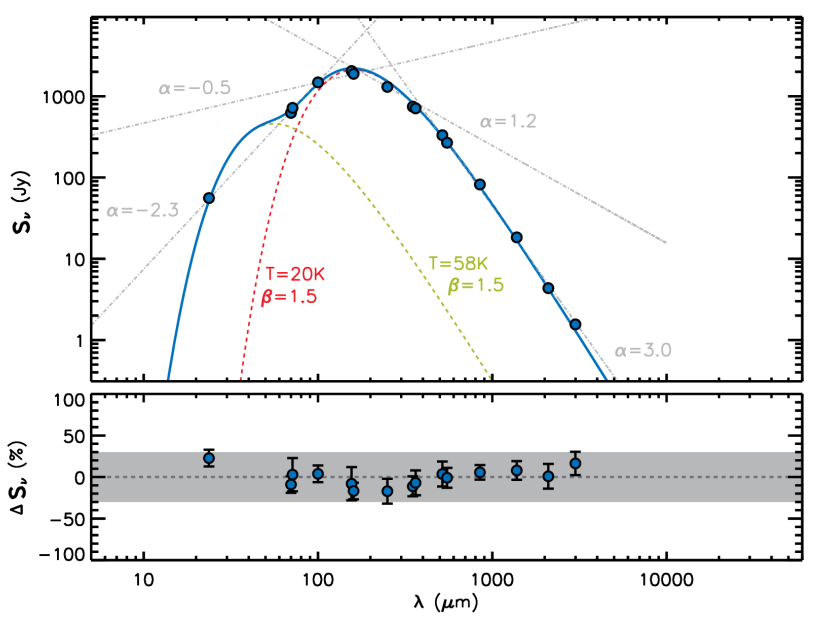

Fig. A.1. Characterization of the SED of M 33 using MBB and powerlaw spectra. The residuals shown in the bottom panel correspond to the MBB fit. The gray shaded area of $\pm 30 \%$ is included to facilitate the reading.

As we mentioned in Sect. 3, our photometric measurements were color corrected. Color corrections are necessary to compare monochromatic fluxes with the models of Popescu et al. (2011) and Groves et al. (2008). In order to select appropriate SED profiles in the immediacy of each data point, we mimicked the observations of M 33 using MBB models and power laws, $S_{v} \propto v^{\alpha}$.

We found that the observations of M33 can be reproduced well with both a two-component modified black body of $\beta=1.5$ and $T=20 \mathrm{~K}$ and $T=57 \mathrm{~K}$ or a combination of four power laws with indices ranging from -2.3 to 3.0 (see Fig. A.1 for details). Then we searched in the tables for MIPS ${ }^{7}$, PACS $^{8}$, SPIRE $^{9}$, and Planck (Planck Collaboration IX 2014) for the listed color corrections that best correspond to the profiles found. For example, for MIPS $24 \mu \mathrm{m}$ we used the value 0.960 tabulated for a power law of index -2.0 (no values are listed for -2.3 ). For the remaining PACS and SPIRE bands we found lower corrections. In the case of Planck data, color corrections are higher with values ranging from 0.963 at $143 \mathrm{GHz}$ to 0.854 at $545 \mathrm{GHz}$.

\section{Appendix B: CMB subtraction}

The angular size of the fluctuations of the cosmic microwave background (CMB) in the line of sight of M 33 is comparable to the size of the galaxy itself (see Fig. B.1). For this reason, the contribution of the CMB to the flux of M33 must be removed

\footnotetext{
7 http://irsa.ipac.caltech.edu/data/SPITZER/docs/mips/ mipsinstrumenthandbook/

8 http://herschel.esac.esa.int/Docs/PACS/html/pacs_om. html

9 http://herschel. esac. esa.int/Docs/SPIRE/html/spire_ om.html
}

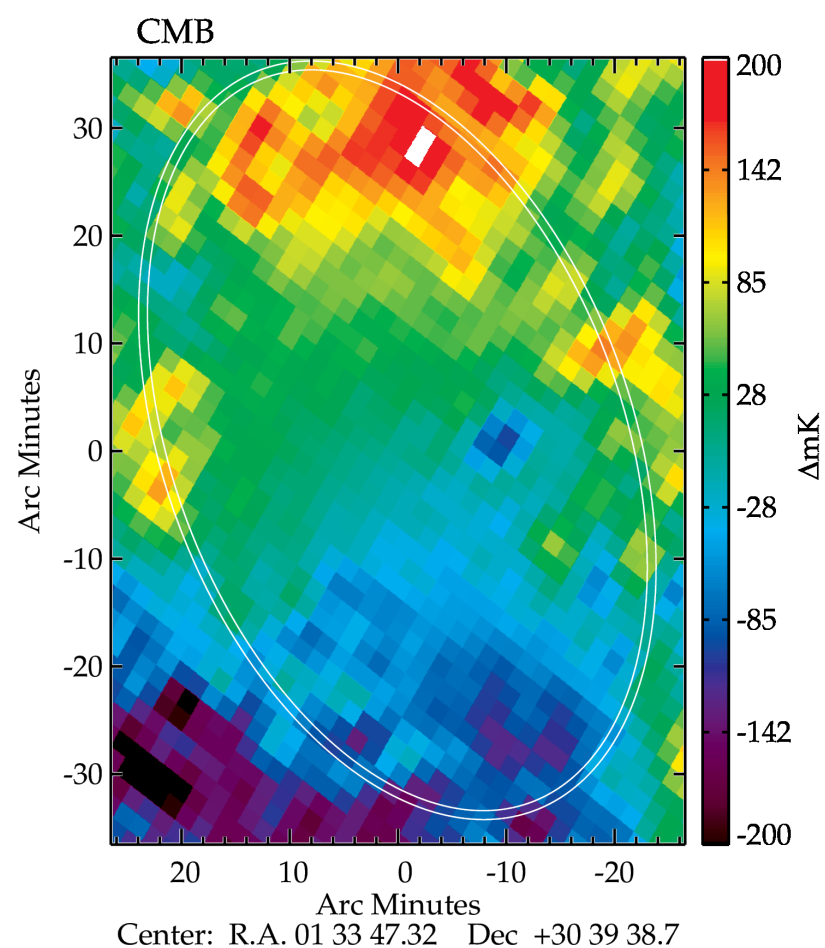

Fig. B.1. Gnomonic projection of the CMB map of Planck in the line of sight of M 33 generated with the SMICA method. The white annulus used to subtract the local background in Fig. 1 was included here for reference. The pixel size is 2 '.6.

on a pixel-by-pixel basis instead of simply assuming a homogeneous background.

Four different CMB maps obtained with different component separation techniques are available in the Planck Legacy Archive (see Table 1 in Planck Collaboration XII 2014). The Spectral Matching Independent Component Analysis method (SMICA), reconstructs the CMB map as a linear combination of all the nine Planck frequency channels harmonically transformed up to a multipole $\ell=4000$ (see Appendix D in Planck Collaboration XII 2014, for details).

Planck Collaboration XXVIII (2015) used the SMICA map to subtract the CMB from M 31, as from a visual inspection of the four methods this appears to be the cleanest map of the CMB in that region. Following these authors, we also made use of the SMICA map to remove the CMB in the line of sight of M 33. The Gnomonic projection of the SMICA map was generated using the same method explained in Sect. 2.7.

\section{Appendix C: Alternative thresholds}

In addition to the threshold of 40 times the background noise used in this paper to separate the SF and the diffuse components (see Sect. 3.1 for details), we also examined the results obtained when we adopted thresholds of 20 (see Fig. C.1) and 60 (see Fig. C.2). As Tables C.1 and C.2 show, the results derived from our modeling does not depend significantly on the value of the threshold used; i.e., independently of the threshold we found i) similar deviations between the models and the observations; ii) similar dust masses; and iii) similar fractions of UV radiation escaping M 33. The main reason is that the emission from the SF component constitutes only a small fraction of the total SED for most wavelengths $(100 \mu \mathrm{m} \lesssim \lambda \lesssim 1 \mathrm{~mm})$. 
I. Hermelo et al.: Millimeter and submillimeter excess emission in M 33 revealed by Planck and LABOCA
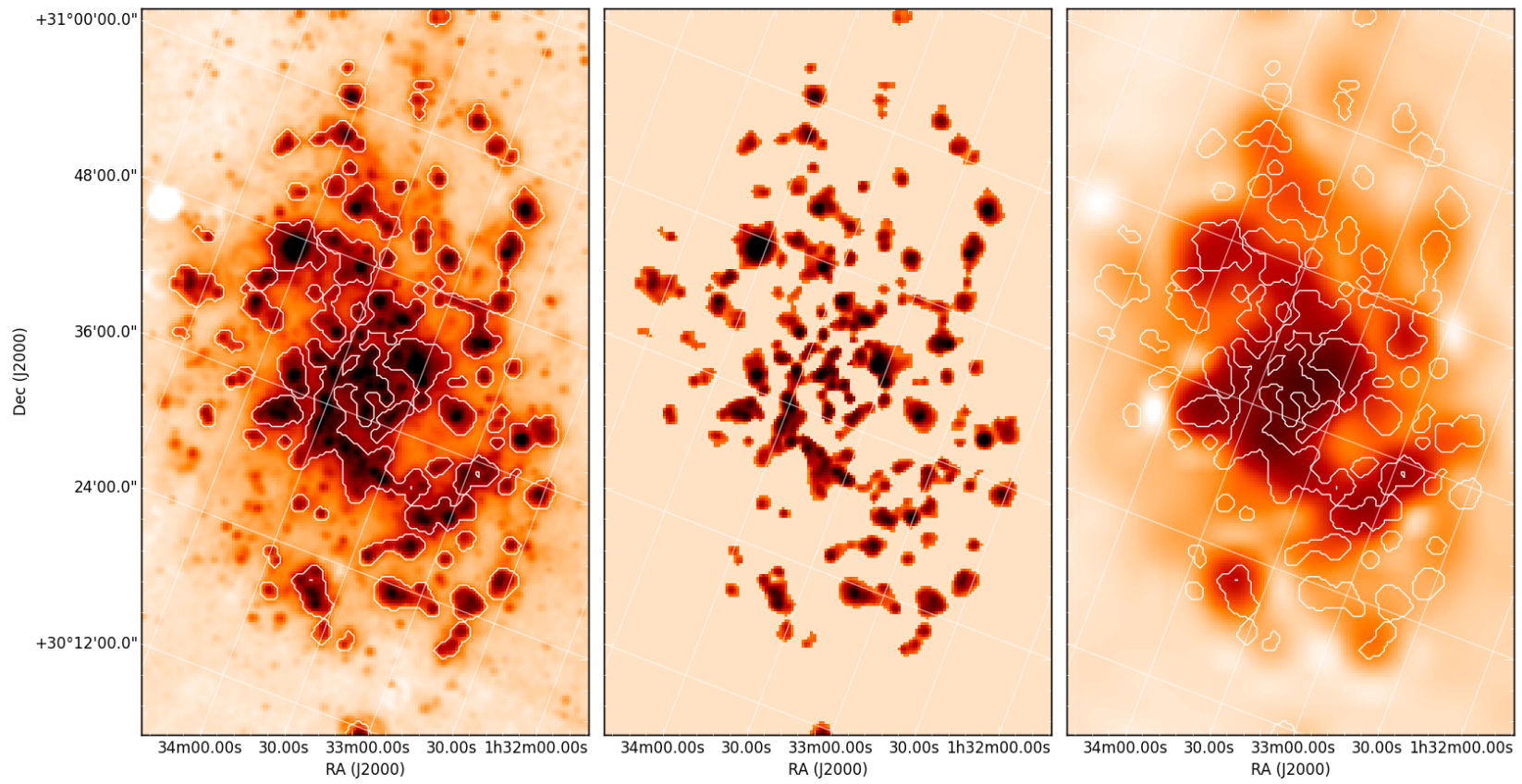

Fig. C.1. Same as Fig. 2 for a threshold of 20 times the background noise.
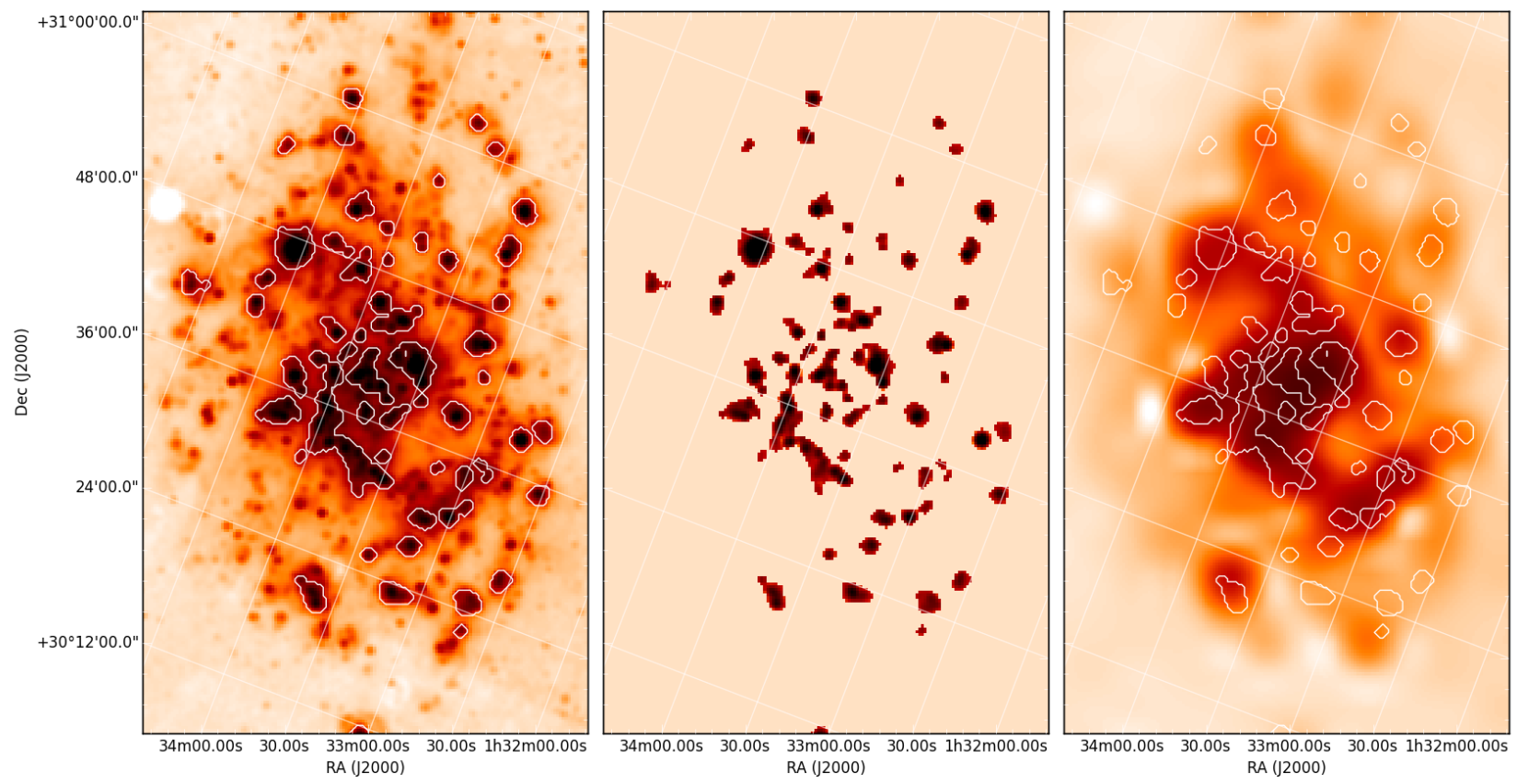

Fig. C.2. Same as Fig. 2 for a threshold of 60 times the background noise. 
Table C.1. Same as Table 2 for a threshold of 20 times the background noise.

\begin{tabular}{|c|c|c|c|c|c|c|}
\hline BAND & $\frac{S_{\mathrm{SF}}^{\mathrm{obs}}}{S_{\mathrm{SF}}^{\bmod }}$ & $\frac{\Delta_{\mathrm{SF}}}{\sqrt{\left(\delta_{\mathrm{SF}}^{\mathrm{obs}}\right)^{2}+\left(\delta_{\mathrm{SF}}^{\mathrm{mod}}\right)^{2}}}$ & $\frac{S_{\mathrm{DIFF}}^{\text {obs }}}{S_{\mathrm{DIFF}}^{\text {mod }}}$ & $\frac{\Delta_{\mathrm{DIFF}}}{\sqrt{\left(\delta_{\mathrm{DIFF}}^{\mathrm{obs}}\right)^{2}+\left(\delta_{\mathrm{DIFF}}^{\mathrm{mod}}\right)^{2}}}$ & $\frac{S_{\mathrm{TOT}}^{\mathrm{obs}}}{S_{\mathrm{TOT}}^{\text {mod }}}$ & $\frac{\Delta_{\mathrm{TOT}}}{\sqrt{\left(\delta_{\mathrm{TOT}}^{\text {obs }}\right)^{2}+\left(\delta_{\mathrm{TOT}}^{\text {mod }}\right)^{2}}}$ \\
\hline GALEX FUV & - & - & - & - & $5.70_{\vee 3.01}^{\wedge 190.39}$ & 5.22 \\
\hline GALEX NUV & - & - & - & - & $4.30_{\vee 3.02}^{\wedge}$ & 7.49 \\
\hline Sloan $u$-band & - & - & - & - & $1.70_{\vee 1.35}^{\wedge} 2.31$ & 2.68 \\
\hline MIPS $24 \mu \mathrm{m}$ & $1.15_{\vee}^{\wedge} 1.33$ & 0.69 & $1.01 \wedge 1.21$ & 0.02 & $1.04 \stackrel{\wedge}{\wedge} 1.23$ & 0.25 \\
\hline PACS $70 \mu \mathrm{m}$ & $0.95 \vee 1.05$ & -0.32 & $1.06_{\vee 0.83}^{\wedge 1.38}$ & 0.20 & $1.02 \stackrel{\wedge}{\wedge 1.23}$ & 0.11 \\
\hline PACS $100 \mu \mathrm{m}$ & $0.95 \wedge 1.10$ & -0.26 & $0.98_{\vee 0.80}^{\wedge} 1.17$ & -0.11 & $0.97 \wedge 1.15$ & -0.15 \\
\hline PACS $160 \mu \mathrm{m}$ & $0.98_{\vee 0.86}^{\wedge}$ & -0.06 & $0.98_{\vee 0.85}^{\wedge}$ & -0.11 & $0.98_{\vee}^{\wedge} 1.08$ & -0.09 \\
\hline SPIRE $250 \mu \mathrm{m}$ & $1.00 \wedge 1.17$ & -0.01 & $0.89 \vee 0.97$ & -0.93 & $0.91 \wedge 1.01$ & -0.76 \\
\hline Planck $350 \mu \mathrm{m}$ & - & - & $1.02 \wedge 1.16$ & 0.11 & $1.02 \wedge 1.16$ & 0.10 \\
\hline SPIRE $350 \mu \mathrm{m}$ & $1.10_{\vee 0.96}^{\wedge}$ & 0.63 & $1.03 \wedge 1.17$ & 0.17 & $1.03 \wedge 1.18$ & 0.23 \\
\hline SPIRE $500 \mu \mathrm{m}$ & $1.29 \wedge 1.56$ & 1.76 & $1.14_{\vee 0.98}^{\wedge 1.33}$ & 0.75 & $1.17 \stackrel{1.37}{\vee 1.00}$ & 1.07 \\
\hline Planck $550 \mu \mathrm{m}$ & - & - & $1.36_{\vee 1.15}^{\wedge 1.58}$ & 1.71 & $1.31_{\vee 1.12}^{\wedge}$ & 1.50 \\
\hline Planck $850 \mu \mathrm{m}$ & - & - & $1.62 \wedge 1.92$ & 2.95 & $1.54 \stackrel{\wedge}{\wedge} 1.84$ & 2.66 \\
\hline LABOCA $870 \mu \mathrm{m}$ & $1.80_{\vee}^{\wedge} 2.21$ & 4.39 & - & - & - & - \\
\hline Planck $1.4 \mathrm{~mm}$ & - & - & $2.11_{\vee 1.74}^{\wedge 2.52}$ & 4.83 & $1.95_{\vee}^{\wedge} 2.33$ & 4.38 \\
\hline Planck $2.1 \mathrm{~mm}$ & - & - & $1.99 \stackrel{2.39}{\vee} 1.63$ & 3.83 & $1.75_{\vee}^{\wedge} 2.08$ & 3.21 \\
\hline Planck $3.0 \mathrm{~mm}$ & - & - & $2.93 \vee 2.43$ & 7.88 & $1.99 \wedge 2.36$ & 4.73 \\
\hline Planck $10 \mathrm{~mm}$ & - & - & - & - & $0.85_{\vee}^{\wedge} 1.00$ & -0.57 \\
\hline $\mathrm{S} 36 \mathrm{~mm}$ & $0.79 \wedge 0.94$ & -1.05 & - & - & $0.79_{\vee 0.69}^{\wedge}$ & -1.05 \\
\hline
\end{tabular}

Table C.2. Same as Table 2 for a threshold of 60 times the background noise.

\begin{tabular}{|c|c|c|c|c|c|c|}
\hline BAND & $\frac{S_{\mathrm{SF}}^{\mathrm{obs}}}{S_{\mathrm{SF}}^{\bmod }}$ & $\frac{\Delta_{\mathrm{SF}}}{\sqrt{\left(\delta_{\mathrm{SF}}^{\mathrm{obs}}\right)^{2}+\left(\delta_{\mathrm{SF}}^{\mathrm{mod}}\right)^{2}}}$ & $\frac{S_{\mathrm{D}}^{\mathrm{obs}}}{S_{\mathrm{DIFF}}^{\bmod }}$ & $\frac{\Delta_{\text {DIFF }}}{\sqrt{\left(\delta_{\text {DIFF }}^{\text {obs }}\right)^{2}+\left(\delta_{\text {DIFF }}^{\text {mod }}\right)^{2}}}$ & $\frac{S_{\mathrm{TOT}}^{\text {obs }}}{S_{\mathrm{TOT}}^{\text {mod }}}$ & $\frac{\Delta_{\mathrm{TOT}}}{\sqrt{\left(\delta_{\mathrm{TOT}}^{\text {obs }}\right)^{2}+\left(\delta_{\mathrm{TOT}}^{\mathrm{mod}}\right)^{2}}}$ \\
\hline GALEX FUV & - & - & - & - & $3.54_{\vee}^{\wedge} 197.88$ & 5.19 \\
\hline GALEX NUV & - & - & - & - & $3.67 \stackrel{\vee}{\vee 2.18}$ & 7.81 \\
\hline Sloan $u$-band & - & - & - & - & $1.64 \vee 2.35$ & 2.66 \\
\hline MIPS $24 \mu \mathrm{m}$ & $1.08_{\vee 0.90}^{\wedge 1.25}$ & 0.37 & $1.02 \wedge 1.29$ & 0.13 & $1.02 \wedge 1.24$ & 0.09 \\
\hline PACS $70 \mu \mathrm{m}$ & $0.99 \wedge 1.12$ & -0.03 & $1.08_{\vee}^{\wedge} 1.52$ & 0.36 & $1.06 \vee 1.36$ & 0.23 \\
\hline PACS $100 \mu \mathrm{m}$ & $1.02 \wedge 1.17$ & 0.09 & $0.97 \vee 1.22$ & -0.13 & $0.98_{\vee}^{\wedge} 1.21$ & -0.09 \\
\hline PACS $160 \mu \mathrm{m}$ & $1.03 \wedge 1.18$ & 0.11 & $0.96_{\vee 0.86}^{\wedge 1.08}$ & -0.15 & $0.98 \wedge 1.09$ & -0.10 \\
\hline SPIRE $250 \mu \mathrm{m}$ & $0.98 \wedge 1.14$ & -0.10 & $0.88_{\vee}^{\wedge} 1.04$ & -0.69 & $0.90_{\vee 0.82}^{\wedge}$ & -0.62 \\
\hline Planck $350 \mu \mathrm{m}$ & - & - & $1.01 \wedge 1.21$ & 0.05 & $1.01 \wedge 1.21$ & 0.03 \\
\hline SPIRE $350 \mu \mathrm{m}$ & $1.06_{\vee 0.91}^{\wedge 1.28}$ & 0.36 & $1.02 \wedge 1.24$ & 0.20 & $1.02 \wedge 1.23$ & 0.11 \\
\hline SPIRE $500 \mu \mathrm{m}$ & $1.21 \vee 1.49$ & 1.19 & $1.14 \vee 1.40$ & 1.05 & $1.15_{\vee 1.04}^{\wedge 1.42}$ & 1.20 \\
\hline Planck $550 \mu \mathrm{m}$ & - & - & $1.32 \wedge 1.63$ & 2.03 & $1.29 \vee 1.59$ & 1.80 \\
\hline Planck $850 \mu \mathrm{m}$ & - & - & $1.57 \stackrel{1}{\vee} 1.97$ & 3.77 & $1.52 \wedge 1.90$ & 3.39 \\
\hline LABOCA $870 \mu \mathrm{m}$ & $1.72 \wedge 2.14$ & 3.62 & - & - & - & - \\
\hline Planck $1.4 \mathrm{~mm}$ & - & - & $2.04 \wedge 2.57$ & 6.02 & $1.92 \wedge 2.42$ & 5.38 \\
\hline Planck $2.1 \mathrm{~mm}$ & - & - & $1.93 \wedge 2.46$ & 4.56 & $1.75_{\vee 1.51}^{\wedge 2.18}$ & 3.57 \\
\hline Planck $3.0 \mathrm{~mm}$ & - & - & $2.86_{\vee 2.47}^{\wedge 3.64}$ & 9.90 & $2.02 \wedge 2.51$ & 5.01 \\
\hline Planck $10 \mathrm{~mm}$ & - & - & - & - & $0.89_{\vee 0.74}^{\wedge} 1.09$ & -0.38 \\
\hline $\mathrm{S} 36 \mathrm{~mm}$ & $0.84_{\vee 0.69}^{\wedge 1.02}$ & -0.75 & - & - & $0.84_{\vee 0.69}^{\wedge}$ & -0.75 \\
\hline
\end{tabular}

